\title{
Cycles in the chamber homology of GL(3)
}

\author{
Anne-Marie Aubert, Samir Hasan and Roger Plymen
}

\begin{abstract}
Let $F$ be a nonarchimedean local field and let $\mathrm{GL}(N)=\mathrm{GL}(N, F)$. We prove the existence of parahoric types for $\operatorname{GL}(N)$. We construct representative cycles in all the homology classes of the chamber homology of GL(3).
\end{abstract}

\section{Introduction}

Let $F$ be a nonarchimedean local field and let $G=\operatorname{GL}(N)=\operatorname{GL}(N, F)$. The enlarged building $\beta^{1} G$ of $G$ is a polysimplicial complex on which $G$ acts properly. We select a chamber $C \subset \beta^{1} G$. This chamber is a polysimplex, the product of an $n$-simplex by a 1 -simplex:

$$
C=\Delta_{n} \times \Delta_{1} .
$$

To this datum we will attach a homological coefficient system, see [13, p.11]. To each simplex $x \in \Delta_{n}$ we attach the representation $\operatorname{ring} R(G(x))$ of the stabilizer $G(x)$, and to each inclusion $x \rightarrow y$ we attach the induction map:

$$
\operatorname{Ind}_{G(x)}^{G(y)}: R(G(x)) \rightarrow R(G(y)) .
$$

This creates the homology of the simplicial set $\Delta_{n}$ with the above coefficient system. The resulting homology groups are denoted $h_{j}(G), 0 \leq j \leq$ $N-1$.

For each point $\mathfrak{s}$ in the Bernstein spectrum $\mathfrak{B}(G)$ (see appendix B) we will select an $\mathfrak{s - t y p e}(J, \tau)$. Here, $J$ denotes a certain compact open subgroup of $G$, and $\tau$ denotes a certain irreducible smooth representation of $J$, see [8, 9, 10].

The following result is due to Bushnell-Kutzko [8, 9, 10].

Theorem 1. Existence of types. Let $\mathfrak{s} \in \mathfrak{B}(G)$. There exists an $\mathfrak{s}$-type $(J, \tau)$. 
Let $\mathfrak{s} \in \mathfrak{B}(G)$. An $\mathfrak{s}$-type $(J, \lambda)$ will be called parahoric if $J$ is a parahoric subgroup of $G$.

Our first result is the following theorem.

Theorem 2. Existence of parahoric types. Let $\mathfrak{s} \in \mathfrak{B}(G)$. Then there exists a parahoric $\mathfrak{s}$-type $\left(J^{\mathfrak{s}}, \tau\right)$.

The parahoric subgroup $J^{\mathfrak{s}}$ only depends on certain invariants attached to $\mathfrak{s}$. For details of these invariants, see appendix D.

In the proof of Theorem 2, we have to call upon several of the technical resources developed by Bushnell-Kutzko.

We now specialize to GL(3). In this article, we will explicitly construct representative cycles in all the homology classes in $h_{0}(G) \oplus h_{1}(G) \oplus h_{2}(G)$ when $G=\mathrm{GL}(3)$. This allows us to compute the chamber homology groups of $\mathrm{GL}(3)$ according to the following formulas:

$$
\mathrm{H}_{\mathrm{ev}}\left(G ; \beta^{1} G\right)=h_{0}(G) \oplus h_{1}(G) \oplus h_{2}(G)=\mathrm{H}_{\mathrm{odd}}\left(G ; \beta^{1} G\right) .
$$

We will demonstrate that each parahoric $\mathfrak{s}$-type $\left(J^{\mathfrak{s}}, \tau\right)$ creates finitely many cycles in $h_{0}(G) \oplus h_{1}(G) \oplus h_{2}(G)$. To prove that all homology classes in $h_{0}(G) \oplus h_{1}(G) \oplus h_{2}(G)$ are thereby accounted for, we invoke the $K$-theory of the reduced $C^{*}$-algebra $\mathcal{A}:=C_{r}^{*}(G)$. The $K$-theory is torsion-free [17].

The abelian groups $\mathrm{H}_{\text {ev/odd }}\left(G ; \beta^{1} G\right)$ and $K_{j}(\mathcal{A})$ admit compatible Bernstein decompositions, see appendix B. This leads, for each $\mathfrak{s} \in \mathfrak{B}(G)$, to the equalities

$$
\operatorname{rank} \mathrm{H}_{\text {ev } / \text { odd }}\left(G ; \beta^{1} G\right)^{\mathfrak{s}}=\operatorname{rank} K_{0}\left(\mathcal{A}^{\mathfrak{s}}\right)=\operatorname{rank} K_{1}\left(\mathcal{A}^{\mathfrak{s}}\right) .
$$

The ranks of the finitely generated abelian groups on the right-hand-side are easily computed (see appendix C).

Theorem 3. Let $G=\mathrm{GL}(3)$, and let $\mathfrak{s}=[M, \sigma]_{G}$. Each parahoric $\mathfrak{s}$-type $\left(J^{\mathfrak{s}}, \tau\right)$ creates finitely many cycles in $h_{0}(G) \oplus h_{1}(G) \oplus h_{2}(G)$, and all homology classes in $h_{0}(G) \oplus h_{1}(G) \oplus h_{2}(G)$ are thereby accounted for. Quite specifically, we have

- if $M=\mathrm{GL}(3)$ then

$$
\mathrm{H}_{\mathrm{ev}}\left(G ; \beta^{1} G\right)^{\mathfrak{s}}=\mathbb{Z}=\mathrm{H}_{\text {odd }}\left(G ; \beta^{1} G\right)^{\mathfrak{s}}
$$

- if $M=\mathrm{GL}(2) \times \mathrm{GL}(1)$ then

$$
\mathrm{H}_{\mathrm{ev}}\left(G ; \beta^{1} G\right)^{\mathfrak{s}}=\mathbb{Z}^{2}=\mathrm{H}_{\text {odd }}\left(G ; \beta^{1} G\right)^{\mathfrak{s}}
$$


- if $M=\mathrm{GL}(1) \times \mathrm{GL}(1) \times \mathrm{GL}(1)$ then

$$
\mathrm{H}_{\mathrm{ev}}\left(G ; \beta^{1} G\right)^{\mathfrak{s}}=\mathbb{Z}^{4}=\mathrm{H}_{\text {odd }}\left(G ; \beta^{1} G\right)^{\mathfrak{s}}
$$

From this point of view, the types for GL(3) exceed their original expectations. Let $\widehat{\mathcal{A}^{\mathfrak{s}}}$ denote the dual of the $C^{*}$-algebra $\mathcal{A}^{\mathfrak{s}}$. This is a compact Hausdorff space. Since $K$-theory for unital $C^{*}$-algebras is compatible with topological $K$-theory of compact Hausdorff spaces, we have

$$
K_{j}\left(\mathcal{A}^{\mathfrak{s}}\right) \cong K^{j}\left(\widehat{\mathcal{A}^{\mathfrak{s}}}\right)
$$

Therefore, the $\mathfrak{s - t y p e}$ also computes the topological $K$-theory of the compact space $\widehat{\mathcal{A}^{\mathfrak{s}}}$. The space $\widehat{\mathcal{A}^{\mathfrak{s}}}$ is precisely the space of all those tempered representations of GL(3) which have inertial support $\mathfrak{s}$.

Sections $4-6$ are devoted to a proof of Theorem 2, and sections $7-9$ are devoted to a proof of Theorem 3

Preliminary work in the direction of Theorem 3 was done with Paul Baum and Nigel Higson, and recorded in [4]. The diagrams in [4] are relevant to the present article. In [4 all computations were in the tame case. We confront here the general case: this is much more technical. We require much detailed information in the theory of types; in particular we need detailed information concerning compact intertwining sets.

We thank the referees for their detailed and constructive comments.

\section{General results on types}

We will collect here some general results on types which will used in the paper. In this section $G$ denotes the group of $F$-points of an arbitrary reductive connected algebraic group $\mathbf{G}$ defined over $F$.

Let $\mathfrak{R}(G)$ denote the category of smooth complex representations of $G$. Recall that, for each irreducible smooth representation $\pi$ of $G$, there exists a Levi subgroup $L$ of a parabolic subgroup $P$ of $G$ and an irreducible supercuspidal representation $\sigma$ of $L$ such that $\pi$ is equivalent to a subquotient of the parabolically induced representation $I_{P}^{G}(\sigma)$. The pair $(L, \sigma)$ is unique up to conjugacy and the inertial class $\mathfrak{s}=[M, \sigma]_{G}$ (see appendix B) is called the inertial support of $\pi$.

We have the standard decomposition (see [5. (2.10)])

$$
\mathfrak{R}(G)=\prod_{\mathfrak{s} \in \mathfrak{B}(G)} \mathfrak{R}^{\mathfrak{s}}(G)
$$


into full sub-categories, where the objects of $\mathfrak{R}^{\mathfrak{s}}(G)$ are those smooth representations of $G$ all of whose irreducible subquotients have inertial support $\mathfrak{s .}$

Let $\mathfrak{s}$ be a point in the Bernstein spectrum of $G$, and let $(J, \tau)$ be an s-type, i.e., $\tau$ is an irreducible smooth representation of an open compact subgroup $J$ of $G$ such that for any irreducible smooth representation $\pi$ of $G$, the restriction of $\pi$ to $J$ contains $\tau$ if and only if $\pi$ is an object of $\mathfrak{R}^{\mathfrak{s}}(G)$, [9, (4.2)]. When $G=\mathrm{GL}(N, F)$, it has been proved [8, 10] that there exists an $\mathfrak{s}$-type for each point $\mathfrak{s}$ in $\mathfrak{B}(G)$.

Proposition 1. Let $K \supset J$ be an open compact subgroup of $G$ such that $\alpha:=\operatorname{Ind}_{J}^{K} \tau$ is irreducible. Then $(K, \alpha)$ is an $\mathfrak{s}$-type.

Proof. Let $\pi$ be an irreducible smooth representation of $G$. Using Frobenius reciprocity, we see that

$$
\operatorname{Hom}_{K}\left(\alpha, \operatorname{Res}_{K}^{G}(\pi)\right)=\operatorname{Hom}_{J}\left(\tau, \operatorname{Res}_{J}^{G}(\pi)\right) .
$$

The result follows from the definition of $\mathfrak{s}$-types.

Let $J, J^{\prime}, K$ be subgroups of $G$ with $J, J^{\prime}$ compact open and $J \subset K$, $J^{\prime} \subset K$. Let $\lambda, \lambda^{\prime}$ be representations of $J, J^{\prime}$ on finite-dimensional vector spaces $V, V^{\prime}$. Let $g \in G$. Then $g J g^{-1} \cap J^{\prime}$ is a subgroup of $J^{\prime}$. We set ${ }^{g} \lambda(x):=\lambda\left(g^{-1} x g\right)$. We define the $g$-intertwining vector space of $\left(\lambda, \lambda^{\prime}\right)$ to be

$$
\mathcal{I}_{g}\left(\lambda, \lambda^{\prime}\right)=\operatorname{Hom}_{g J g^{-1} \cap J^{\prime}}\left({ }^{g} \lambda, \lambda^{\prime}\right) .
$$

We will write $\mathcal{I}_{g}(\lambda)=\mathcal{I}_{g}(\lambda, \lambda)$.

Definition 1. (1) We say that $g$ intertwines $\lambda$ if $\mathcal{I}_{g}(\lambda) \neq 0$. The $K$ intertwining set of $\lambda$ is

$$
\mathcal{I}_{K}(\lambda)=\left\{g \in K: \mathcal{I}_{g}(\lambda) \neq 0\right\} \subset K .
$$

(2) We say that $g$ intertwines $\lambda$ and $\lambda^{\prime}$ if $\mathcal{I}_{g}\left(\lambda, \lambda^{\prime}\right) \neq 0$. The $K$-intertwining set of $\lambda$ and $\lambda^{\prime}$ is

$$
\mathcal{I}_{K}\left(\lambda, \lambda^{\prime}\right)=\left\{g \in K: \mathcal{I}_{g}\left(\lambda, \lambda^{\prime}\right) \neq 0\right\} \subset K .
$$

In [9, 10, 8], the results centre around identification of the $G$-intertwining set $I_{G}(\lambda)$. In our applications, we shall need only the $K$-intertwining set $I_{K}(\lambda)$ where $K$ is compact.

In order to study the induced representations and their decomposition into irreducible constituents, we need to use the Mackey formulas repeatedly. 
We assume now that $K$ is open compact. Then $J, J^{\prime}$ have finite index in $K$. We have the Mackey formula:

$$
\operatorname{Hom}_{K}\left(\operatorname{Ind}_{J}^{K}(\lambda), \operatorname{Ind}_{J^{\prime}}^{K}\left(\lambda^{\prime}\right)\right) \cong \bigoplus \mathcal{I}_{x}\left(\lambda, \lambda^{\prime}\right)
$$

with $x \in J \backslash K / J^{\prime}$. If $\lambda=\lambda \cong \lambda^{\prime}$ then we set $\mathcal{I}_{g}(\lambda)=\mathcal{I}_{g}\left(\lambda, \lambda^{\prime}\right)$ and we then have the isomorphism of $\mathbb{C}$-vector spaces

$$
\operatorname{End}_{K}\left(\operatorname{Ind}_{J}^{K}(\lambda)\right) \cong \bigoplus \mathcal{I}_{x}(\lambda)
$$

with $x \in J \backslash K / J$.

The following is an immediate consequence: we will use this result repeatedly.

Proposition 2. If $\mathcal{I}_{K}(\lambda)=J$ then $\operatorname{Ind}_{J}^{K}(\lambda)$ is irreducible.

We will use the following immediate result.

Proposition 3. If $\operatorname{Ind}_{J}^{K}(\lambda)$ and $\operatorname{Ind}_{J^{\prime}}^{K}\left(\lambda^{\prime}\right)$ are irreducible, then

$$
\operatorname{Ind}_{J}^{K}(\lambda) \cong \operatorname{Ind}_{J^{\prime}}^{K}\left(\lambda^{\prime}\right) \Longleftrightarrow \mathcal{I}_{K}\left(\lambda, \lambda^{\prime}\right)=J y J^{\prime}
$$

for some element $y$.

Proposition 4. Let $\left(J^{\mathfrak{s}}, \tau^{\mathfrak{s}}\right)$ be an $\mathfrak{s}$-type, $\left(J^{\mathfrak{s}^{\prime}}, \tau^{\mathfrak{s}^{\prime}}\right)$ be a $\mathfrak{s}^{\prime}$-type with $\mathfrak{s}$, $\mathfrak{s}^{\prime}$ in $\mathfrak{B}(G), \mathfrak{s} \neq \mathfrak{s}^{\prime}$. Let $J$ be a compact open subgroup of $G$ such that $J^{\mathfrak{s}} \subset J$, $J^{\mathfrak{s}^{\prime}} \subset J$. Then we have

$$
\operatorname{dim}_{\mathbb{C}} \operatorname{Hom}_{J}\left(\operatorname{Ind}_{J^{\mathfrak{s}}}^{J} \tau^{\mathfrak{s}}, \operatorname{Ind}_{J^{\mathfrak{s}^{\prime}}}^{J} \tau^{\mathfrak{s}^{\prime}}\right)=0 .
$$

Proof. From the Mackey formula (3) , it is equivalent to prove that $\mathcal{I}_{J}\left(\tau^{\mathfrak{s}}, \tau^{\mathfrak{s}^{\prime}}\right)=$ 0 . The proof of the equivalence of (i) and (ii) of [9, Theorem 9.3.a] shows that $\mathfrak{s}=\mathfrak{s}^{\prime}$ if and only if $\mathcal{I}_{G}\left(\tau^{\mathfrak{s}}, \tau^{\mathfrak{s}^{\prime}}\right) \neq 0$. The result follows.

Let $J$ be a compact open subgroup of $G$ and $(\tau, \mathcal{W})$ be an irreducible smooth representation of $J$. Let $\left(\tau^{\vee}, \mathcal{W}^{\vee}\right)$ be the contragredient representation of $(\tau, \mathcal{W})$.

For any subgroup $K$ of $G$, let $\mathcal{H}(K, \tau)$ denote the space of compactly supported functions $f: K \rightarrow \operatorname{End}_{\mathbb{C}}\left(\mathcal{W}^{\vee}\right)$ such that $f\left(j_{1} k j_{2}\right)=\tau^{\vee}\left(j_{1}\right) f(k) \tau^{\vee}\left(j_{2}\right)$, for any $j_{i} \in J, k \in K$. The standard convolution operation gives $\mathcal{H}(K, \tau)$ the structure of an associative unital $\mathbb{C}$-algebra.

Let $M$ be a Levi subgroup of $G$, and let $\left(J_{M}, \tau_{M}\right)$ be a $\mathfrak{t}$-type, with $\mathfrak{t}:=[M, \sigma]_{M}$ a (supercuspidal) point of the Bernstein spectrum of $M$.

We recall from [9, Definition 8.1] that the pair $(J, \tau)$ is a $G$-cover of $\left(J_{M}, \tau_{M}\right)$ if $J \cap M=J_{M}$ and $\tau_{\mid J_{M}} \cong \tau_{M}$, and if the following conditions hold for every parabolic subgroup $P$ of $G$ with Levi subgroup $M$ : 
(1) $(J, \tau)$ it is decomposed with respect to $(M, P)$, that is, $J$ admits the Iwahori decomposition:

$$
J=J \cap U \cdot J_{M} \cdot J \cap \bar{U}
$$

and the groups $J \cap U, J \cap \bar{U}$ are both contained in the kernel of $\tau$ (here $U, \bar{U}$ denote the unipotent radicals of $P$ and of its opposite parabolic subgroup, respectively),

(2) there exists an invertible element of $\mathcal{H}(G, \tau)$ supported on a double coset $J z_{P} J$, where $z_{P}$ is a central element in $M$, which is strongly $(P, J)$-positive in the sense of [9, Definition (6.16)].

The group $\Psi(M)$ of unramified quasicharacters of $M$ has the structure of a complex torus. The action (by conjugation) of $\mathrm{N}_{G}(M)$ on $M$ induces an action of $W(M):=\mathrm{N}_{G}(M) / M$ on $\mathfrak{B}(M)$. Let $W_{\mathfrak{t}}$ denote the stabilizer of $\mathfrak{t}=[M, \sigma]_{M}$ in $W(M)$. Thus $W_{\mathfrak{t}}=N_{\mathfrak{t}} / M$, where

$$
\mathrm{N}_{\mathfrak{t}}=\left\{n \in \mathrm{N}_{G}(M):{ }^{n} \sigma \cong \nu \sigma, \text { for some } \nu \in \Psi(M)\right\}
$$

denotes the $\mathrm{N}_{G}(M)$-normalizer of $\mathfrak{t}$.

We will need the following Proposition which gives a bound for the compact intertwining.

Proposition 5. [1] We assume here that $G=\operatorname{GL}(N, F)$. Let $M$ be a Levi subgroup of $G$, let $(J, \tau)$ be a $G$-cover of a $\mathfrak{t}$-type, with $\mathfrak{t}=[M, \sigma]_{M}$ a point of the Bernstein spectrum of $M$, and let $K$ be a compact subgroup of $G$ which contains $J$. Let $t$ denote the number of double classes $J \backslash K / J$ which intertwine $\tau$. Then

$$
t \leq\left|W_{\mathfrak{t}}\right|
$$

Proof. It is a classical result that $t$ is bounded by the dimension of $\mathcal{H}(K, \tau)$. The hypotheses on the the supercuspidal representation $\sigma$ which are listed in [11, $\S 1.3]$ are identical to those listed in [9, (5.5)]. Since $G=\mathrm{GL}(N, F)$, it follows from [9, Comments (b) and (d) on (5.5)] that these hypotheses are satisfied, and so we can apply [11, Theorem 1.5(ii)]. We infer that

$$
\operatorname{dim}_{\mathbb{C}} \mathcal{H}(K, \tau) \leq\left|W_{\mathfrak{t}}\right| .
$$




\section{Chamber homology groups}

Let $\mathfrak{o}_{F}$ denote the ring of integers of $F$, let $\varpi=\varpi_{F}$ be a uniformizer in $F$, and $\mathfrak{p}_{F}=\varpi_{F} \mathfrak{o}_{F}$ denote the maximal ideal of $\mathfrak{o}_{F}$. We set

$$
\Pi=\Pi_{N}=\left(\begin{array}{cc}
0 & \mathrm{I}_{N-1} \\
\varpi & 0
\end{array}\right) .
$$

Let $s_{0}, s_{1}, \ldots, s_{N-1}$ denote the standard involutions in $G$ : $s_{i}$ denote the matrix in $G$ of the transposition $i \leftrightarrow i+1$, that is,

$$
s_{i}=\left(\begin{array}{llll}
\mathrm{I}_{i-1} & & & \\
& 0 & 1 & \\
& 1 & 0 & \\
& & & \mathrm{I}_{N-i-1}
\end{array}\right),
$$

for every $i \in\{1, \ldots, N-1\}$, and $s_{0}=\Pi s_{1} \Pi^{-1}$.

The finite Weyl group is $W_{0}=<s_{1}, s_{2}, \ldots, s_{N-1}>$, and the affine Weyl group is given by $W=<s_{0}, s_{1}, \ldots, s_{N-1}>$.

We set

$$
\mathcal{R}(g)=\Pi^{-1} g \Pi
$$

with $g \in G$, so that $\mathcal{R}^{N}=1$.

We will use repeatedly, and without further comment, the fact that induction commutes with conjugation: in particular conjugation by $\operatorname{Ad}^{i}$, $1 \leq i \leq N-1$. We will use this in the following form:

$$
\mathcal{R}^{-1}\left(\operatorname{Ind}_{\mathcal{R} H}^{\mathcal{R} G}(\mathcal{R} \alpha)\right) \cong \operatorname{Ind}_{H}^{G}(\alpha) .
$$

Note that

$$
\mathcal{R}\left(s_{i}\right)=s_{i+1}, \quad \text { with } i=0,1, \ldots, N-1 \quad \bmod N .
$$

The extended affine Weyl group is given by $\widetilde{W}=W \rtimes<\Pi>$. We observe that

$$
\widetilde{W} \cap \operatorname{GL}\left(N, \mathfrak{o}_{F}\right)=W_{0}
$$

The standard Iwahori subgroup is

$$
I=\left(\begin{array}{cccc}
\mathfrak{o}_{F}^{\times} & \mathfrak{o}_{F} & \cdots & \mathfrak{o}_{F} \\
\mathfrak{p}_{F} & \ddots & \ddots & \vdots \\
\vdots & \ddots & \ddots & \mathfrak{o}_{F} \\
\mathfrak{p}_{F} & \cdots & \mathfrak{p}_{F} & \mathfrak{o}_{F}^{\times}
\end{array}\right)
$$

Let $A$ be the apartment attached to the diagonal torus and let $\Delta$ denote the unique chamber of of $A$ which is stabilized by $<\Pi>I$. We index the vertices $L_{0}, L_{1}, \ldots, L_{N-1}$ of $\Delta$ in such a way that 
- $s_{i} \Delta$ is the unique chamber of $A$ which is adjacent to $\Delta$ and such that $s_{i} \Delta \cap \Delta$ is the $(N-2)$-simplex $\left\{L_{0}, \ldots, L_{N-1}\right\} \backslash\left\{L_{i}\right\}$;

- $\mathcal{R}\left(L_{i}\right)=L_{i+1}$ with $i=0,1, \ldots, N-1 \bmod N$.

The $L_{i}$ are the maximal standard parahoric subgroups of $G$,

$$
L_{i}=I<s_{0}, s_{1}, \ldots, s_{i-1}, s_{i+1}, \ldots, s_{N-1}>I=\mathcal{R}^{i}\left(L_{0}\right),
$$

and $L_{0}=\mathrm{GL}\left(N, \mathfrak{o}_{F}\right)$.

The stabilizers of the facets of dimension $N-1$ of $\Delta$ are $J_{0}, J_{1}, \ldots, J_{N-1}$, where

$$
J_{i}=I<s_{i}>I .
$$

Each parahoric subgroup of $G$ is defined by a facet of the building and the standard parahoric subgroups are the

$$
J_{S}=I<s_{j}: j \in S>I,
$$

where $S$ is any subset of $\{0,1, \ldots, N-1\} \bmod . N$, [19, p. 118].

Hence, $I=J_{\emptyset}, J_{i}=J_{\{i\}}, L_{i}=J_{\{0,1, \ldots, i-1, i+1, \ldots, N-1\}}$.

The enlarged building $\beta^{1} G$ is labellable, that is, there exists a simplicial map $\ell: \beta^{1} G \rightarrow \Delta$, which preserves the dimensions of the simplices. The labelling is unique, up to the automorphisms of $\Delta$. It allows us to fix an orientation of the simplices: one defines an incidence number $\langle\eta: \sigma\rangle$ between an arbitrary facet $\eta=\left(\eta_{0}, \ldots, \eta_{i-1}\right)$ of dimension $i$ and any facet $\sigma=\left(\sigma_{0}, \ldots, \sigma_{i}\right)$ of dimension $i+1$ which contains $\eta$, as follows

$$
<\eta: \sigma>=(-1)^{i} \text { if }\left\{\ell\left(\eta_{0}\right), \ldots, \ell\left(\eta_{i-1}\right)\right\} \backslash\left\{\ell\left(\sigma_{0}\right), \ldots, \ell\left(\sigma_{i}\right)\right\}=i .
$$

The chamber homology groups are obtained by totalizing the bicomplex $C_{* *}$

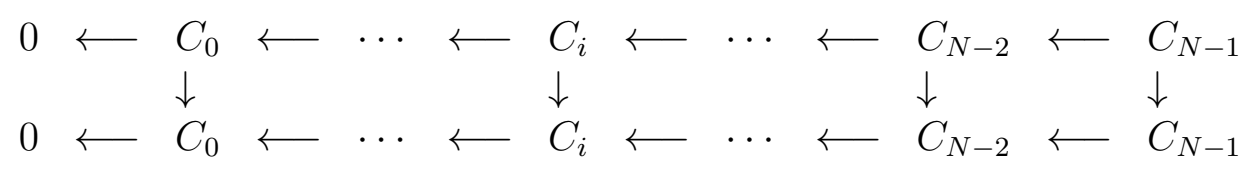

in which the chains are as follows:

$$
C_{i}=\bigoplus_{\substack{S \subset\{0,1, \ldots, N-1\} \\|S|=N-1-i}} R\left(J_{S}\right)
$$

and each vertical map is given by $1-\mathrm{Ad} \Pi$. In particular, we have 
- $C_{0}=R\left(L_{0}\right) \oplus R\left(L_{1}\right) \oplus \cdots \oplus R\left(L_{N-1}\right)$,

- $C_{N-2}=R\left(J_{0}\right) \oplus R\left(J_{1}\right) \oplus \cdots \oplus R\left(J_{N-1}\right)$,

- $C_{N-1}=R(I)$.

We will write an arbitrary element $v$ in $C_{i}$ as a $\left(\begin{array}{c}N \\ i\end{array}\right)$-uple $[\eta]$. Once an orientation has been chosen, the differentials are as follows: if $v \in C_{i}$ then

$$
\partial(v)=\sum_{\substack{\eta \subset \sigma \\ \operatorname{dim} \eta=i}}(-1)^{\langle\eta: \sigma\rangle} \operatorname{Ind}_{G(\sigma)}^{G(\eta)}[\eta] \in C_{i-1}
$$

In particular:

- if $v \in C_{N-1}$ then $\partial(v)=\left(\operatorname{Ind}_{I}^{J_{0}}(v), \operatorname{Ind}_{I}^{J_{1}}(v), \ldots, \operatorname{Ind}_{I}^{J_{N-1}}(v)\right)$,

- if $v \in C_{0}$ then $\partial(v)=0$.

When $G=\mathrm{GL}(3)$, if $v=\left(v_{0}, v_{1}, v_{2}\right) \in C_{1}$ then $\partial(v)$ equals

$$
\left(\operatorname{Ind}_{J_{2}}^{L_{0}}\left(v_{2}\right)-\operatorname{Ind}_{J_{1}}^{L_{0}}\left(v_{1}\right), \operatorname{Ind}_{J_{0}}^{L_{1}}\left(v_{0}\right)-\operatorname{Ind}_{J_{2}}^{L_{1}}\left(v_{2}\right),-\operatorname{Ind}_{J_{0}}^{L_{2}}\left(v_{0}\right)+\operatorname{Ind}_{J_{1}}^{L_{2}}\left(v_{1}\right)\right),
$$

and, in the chain complex

$$
0 \longleftarrow C_{0} \longleftarrow C_{1} \longleftarrow \longleftarrow C_{2} \longleftarrow 0
$$

we have that $v$ is a 1-cycle if and only if

$$
\operatorname{Ind}_{J_{2}}^{L_{0}}\left(v_{2}\right)=\operatorname{Ind}_{J_{1}}^{L_{0}}\left(v_{1}\right), \operatorname{Ind}_{J_{0}}^{L_{1}}\left(v_{0}\right)=\operatorname{Ind}_{J_{2}}^{L_{1}}\left(v_{2}\right), \operatorname{Ind}_{J_{0}}^{L_{2}}\left(v_{0}\right)=\operatorname{Ind}_{J_{1}}^{L_{2}}\left(v_{1}\right),
$$

i.e., if and only if the 1 -chain $\left(v_{0}, v_{1}, v_{2}\right)$ is vertex compatible. Note that a true representation in $R(I)$ can never be a 2-cycle; on the other hand, each 0 -chain is a 0 -cycle.

When we totalize the bicomplex we obtain the chain complex

$0 \longleftarrow C_{0} \longleftarrow C_{0} \oplus C_{1} \longleftarrow \cdots \longleftarrow C_{i-1} \oplus C_{i} \longleftarrow C_{i} \oplus C_{i+1} \longleftarrow \cdots \longleftarrow C_{N-1} \longleftarrow 0$

Definition 2. The homology groups of this totalized complex are the chamber homology groups, as in [4].

To each point $\mathfrak{s} \in \mathfrak{B}(G)$ we will associate a sub-bicomplex $C_{* *}(\mathfrak{s})$ :

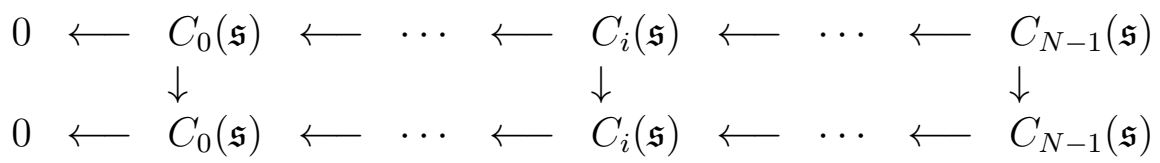


in which each vertical differential is 0. By an invariant chain we shall mean a chain invariant under Ad $\Pi$.

Let $\mathfrak{s}$ be a point in $\mathfrak{B}(G)$ with $\mathfrak{s}=[M, \sigma]_{G}$. We recall that $W(M)$ denotes the group $\mathrm{N}_{G}(M) / M$. We take for $M$ a standard Levi subgroup of $G$, isomorphic to $\operatorname{GL}\left(N_{1}\right) \times \cdots \times \operatorname{GL}\left(N_{r}\right)$, with $\left(N_{1} \geq N_{2} \geq \cdots \geq N_{r}\right)$ a partition of $N$.

Given a point $\mathfrak{s} \in \mathfrak{B}(G)$, fix an $\mathfrak{s}$-type $(J, \tau)$. Such types exist [8, 9, 10]. There exists a parahoric subgroup $J^{\mathfrak{s}}$ containing $J$ such that $\left(J^{\mathfrak{s}}, \alpha:=\operatorname{Ind}_{J}^{J^{\mathfrak{s}}} \tau\right)$ is also an $\mathfrak{s}$-type (see Theorems 4, 5, 6).

Then

- induce (if possible) each element in the orbit $W(M) \cdot \alpha$ to the standard parahoric subgroups containing $J^{\mathfrak{s}}$, and rotate, i.e., apply $\mathcal{R}, \ldots, \mathcal{R}^{N-1}$,

- take the free abelian groups generated by all the irreducible components which arise in this way.

Each of our sub-complexes $C_{* *}(\mathfrak{s})$ will come from some or all of this data. All the chain groups in $C_{* *}(\mathfrak{s})$ are finitely generated free abelian groups and comprise invariant chains. The homology groups of the chain complex

$$
0 \longleftarrow C_{0}(\mathfrak{s}) \longleftarrow C_{1}(\mathfrak{s}) \longleftarrow \cdots \longleftarrow C_{N-1}(\mathfrak{s}) \longleftarrow 0
$$

will be denoted $h_{*}(\mathfrak{s})$. We call this the little complex.

When we totalize the associated bicomplex $C_{* *}(\mathfrak{s})$ we obtain the chain complex

$0 \longleftarrow C_{0}(\mathfrak{s}) \longleftarrow \cdots \longleftarrow C_{i-1}(\mathfrak{s}) \oplus C_{i}(\mathfrak{s}) \longleftarrow C_{i}(\mathfrak{s}) \oplus C_{i+1}(\mathfrak{s}) \longleftarrow \cdots \longleftarrow C_{N-1}(\mathfrak{s}) \longleftarrow 0$

The following lemma will speed up our calculations.

Lemma 1. The homology groups $H_{*}(\mathfrak{s})$ of this complex are given by

$$
\begin{gathered}
H_{0}(\mathfrak{s})=h_{0}(\mathfrak{s}), \quad H_{N}(\mathfrak{s})=h_{N-1}(\mathfrak{s}) \\
H_{i+1}(\mathfrak{s})=h_{i}(\mathfrak{s}) \oplus h_{i+1}(\mathfrak{s}), \quad 0 \leq i \leq N-2 \\
H_{\mathrm{eV}}(\mathfrak{s})=h_{0}(\mathfrak{s}) \oplus h_{1}(\mathfrak{s}) \oplus \cdots \oplus h_{N-1}(\mathfrak{s})=H_{\text {odd }}(\mathfrak{s})
\end{gathered}
$$

The even (resp. odd) chamber homology is precisely the total homology of the little complex.

Proof. This is a direct consequence of the fact that each vertical differential in the bicomplex $C_{* *}(\mathfrak{s})$ is 0 . 


\section{Lattice chains and lattice sequences}

Let $V$ be an $F$-vector space of dimension $N$. We recall from [10, Def. 2.1] that a lattice sequence is a function $\Lambda$ from $\mathbb{Z}$ to the set of $\mathfrak{o}_{F}$-lattices in $V$ such that

- $i \geq j$ implies $\Lambda(i) \leq \Lambda(j)$;

- there exists $e=e(\Lambda) \in \mathbb{Z}, e \geq 1$, such that $\Lambda(i+e)=\mathfrak{p}_{F} \Lambda(i)$ for any $i \in \mathbb{Z}$.

The integer $e$ is uniquely determined, and is called the period of $\Lambda$. We have $e \leq N$.

A lattice sequence which is injective as a function is called strict. We will put

$$
\mathfrak{a}_{n}(\Lambda):=\{a \in A: a \Lambda(m) \subset \Lambda(m+n), m \in \mathbb{Z}\}, \quad n \in \mathbb{Z} .
$$

The concept of lattice sequence generalizes the notion of lattice chain: as defined in [8, (1.11)], a lattice chain in $V$ is a set $\mathcal{L}=\left\{L_{i}: i \in \mathbb{Z}\right\}$ of $\mathfrak{o}_{F}$-lattices $L_{i}$ in $V$ such that

- $L_{i} \supset L_{i+1}, L_{i} \neq L_{i+1}$, for any $i \in \mathbb{Z}$;

- there exists $e=e(\mathcal{L}) \in \mathbb{Z}$ such that $L_{i+e}=\mathfrak{p}_{F} L_{i}$, for any $i \in \mathbb{Z}$.

The integer $e$ is uniquely determined, and is called the period of $\mathcal{L}$.

Let $k_{F}$ denote the residue field of $F$. For each $i$, the quotient $L_{i} / L_{i+1}$ is a $k_{F}$-vector space. Write

$$
d_{i}=d_{i}(\mathcal{L}):=\operatorname{dim}_{k_{F}}\left(L_{i} / L_{i+1}\right)
$$

The function $d(\mathcal{L}): i \mapsto d_{i}, i \in \mathbb{Z}$, is periodic of period dividing $e$, and we have

$$
\sum_{i=0}^{e-1} d_{i}=N .
$$

To each lattice chain $\mathcal{L}$ is attached a strict lattice sequence $\Lambda_{\mathcal{L}}$ defined by $\Lambda_{\mathcal{L}}(i):=L_{i}$, for $i \in \mathbb{Z}$. In the opposite direction, to each lattice sequence $\Lambda$ is attached a lattice chain $\mathcal{L}_{\Lambda}$ defined by

$$
\mathcal{L}_{\Lambda}:=\{\Lambda(i): i \in \mathbb{Z}\}
$$

As in [10, §2.6], we extend a lattice sequence $\Lambda$ to a function on the real line $\mathbb{R}$ by setting

$$
\Lambda(x):=\Lambda(\lceil x\rceil), \quad x \in \mathbb{R},
$$


where $\lceil x\rceil$ is the integer defined by the relation $\lceil x\rceil-1<x \leq\lceil x\rceil$.

Let $\Lambda$ be a lattice sequence in $V$ and let $m$ be a positive integer. Then the function $m \Lambda$ from $\mathbb{Z}$ to the set of $\mathfrak{o}_{F}$-lattices in $V$ defined by

$$
(m \Lambda)(i):=\Lambda(i / m), \quad \text { for any } i \in \mathbb{Z}
$$

is a lattice sequence in $V$ with period $m e(\Lambda)$, and we have

$$
(m \Lambda)(i)= \begin{cases}\Lambda(i / m) & \text { if } m \text { divides } i \\ \Lambda(1+[i / m]) & \text { otherwise }\end{cases}
$$

and $(m \Lambda)(x)=\Lambda(x / m)$, for all $x \in \mathbb{R}$ (see [10, Prop. 2.7]).

If we have a lattice sequence $\Lambda$ in $V$ and an integer $t$, we can define a lattice chain $\Lambda+t$ by

$$
(\Lambda+t)(i):=\Lambda(i+t), \text { for any } i \in \mathbb{Z}
$$

Let $m$ be a positive integer, and let $V^{1}, V^{2}, \ldots, V^{m}$ be $m$ finite-dimensional $F$-vector spaces. Let $\Lambda^{1}, \Lambda^{2}, \ldots, \Lambda^{m}$ be $m$ lattices sequences in $V$, with periods $e_{1}, e_{2}, \ldots, e_{m}$, respectively. We denote by $\Lambda=\Lambda^{1} \oplus \cdots \oplus \Lambda^{m}$ the direct sum of $\Lambda^{1}, \ldots, \Lambda^{m}$ : we recall from [10, §2.8] that $\Lambda$ is defined by $\Lambda(e x)=\Lambda^{1}\left(e_{1} x\right) \oplus \cdots \oplus \Lambda^{m}\left(e_{m} x\right), \quad$ for each $x \in \mathbb{R}$, where $e=\operatorname{lcm}\left\{e_{1}, \ldots, e_{m}\right\}$

The following example occurs in the construction of [10, §7.2]. See also [10. Example 2.8].

Example 1. We assume given $m$ lattice chains $\mathcal{L}^{1}, \mathcal{L}^{2}, \ldots, \mathcal{L}^{m}$ in $V^{1}, V^{2}$, $\ldots, V^{m}$, respectively, of same period $e$. We define a lattice chain

$$
\mathcal{L}=\left\{L_{i}: i \in \mathbb{Z}\right\}
$$

in $V$ of period me by setting

$$
L_{m j+k}:=L_{j}^{1} \oplus L_{j}^{2} \oplus \cdots \oplus L_{j}^{m-k} \oplus L_{j+1}^{m-k+1} \oplus \cdots \oplus L_{j+1}^{m},
$$

any $j \in \mathbb{Z}$ and $0 \leq k \leq m-1$. Using (13), (14), we obtain

$$
\Lambda_{\mathcal{L}}=\left(m \Lambda^{1}-m+1\right) \oplus \cdots \oplus\left(m \Lambda^{m-k}-k\right) \oplus \cdots \oplus\left(m \Lambda^{m-1}-1\right) \oplus m \Lambda^{m}
$$




\subsection{Addition of lattice chains}

Let $A:=\operatorname{End}_{F}(V)$ and let $E / F$ be a subfield of $A$. We denote by $\mathfrak{o}_{E}$ the discrete valuation ring in $E$, by $k_{E}$ its residue field, and by $e(E \mid F)$ the ramification degree of $E / F$.

Let $V^{1}, V^{2}, \ldots, V^{m}$ be $m$ finite-dimensional $F$-vector spaces of dimensions $N_{1}, N_{2}, \ldots, N_{m}$, respectively. We assume that the field $E$ preserves the spaces $V^{i}$. We may consider each $V^{l}$ as a $E$-vector space of dimension $N_{l} /[E: F]$.

Let $\mathcal{L}^{1}, \mathcal{L}^{2}, \ldots, \mathcal{L}^{m}$ be $m \mathfrak{o}_{E}$-lattice chains in the $E$-vector spaces $V^{1}, V^{2}$, $\ldots, V^{m}$, respectively, of period $e_{1}^{\prime}, e_{2}^{\prime}, \ldots, e_{m}^{\prime}$, respectively.

\subsubsection{First addition procedure}

We define first an $\mathfrak{o}_{E}$-lattice chain $\mathcal{L}^{1}+\mathcal{L}^{2}=\left\{L_{j}^{[1,2]}: j \in \mathbb{Z}\right\}$ in $V^{1} \oplus V^{2}$ of period $e_{1}^{\prime}+e_{2}^{\prime}$ by

$$
L_{i}^{[1,2]}:= \begin{cases}L_{0}^{1} \oplus L_{i}^{2}, & \text { if } 0 \leq i \leq e_{2}^{\prime}-1 \\ L_{i-e_{2}^{\prime}}^{1} \oplus L_{e_{2}^{\prime}}^{2}, & \text { if } e_{2}^{\prime} \leq i \leq e_{1}^{\prime}+e_{2}^{\prime}-1 .\end{cases}
$$

Then let $\mathcal{L}^{1}+\mathcal{L}^{2}+\mathcal{L}^{3}=\left\{L_{i}^{[1,3]}: i \in \mathbb{Z}\right\}$ be the $\mathfrak{o}_{E}$-lattice chain $\left(\mathcal{L}^{1}+\mathcal{L}^{2}\right)+\mathcal{L}^{3}$ (which is the same as $\mathcal{L}^{1}+\left(\mathcal{L}^{2}+\mathcal{L}^{3}\right)$ ). By applying (16) to the two $\mathfrak{o}_{E}$-lattice chains $\mathcal{L}^{1}+\mathcal{L}^{2}$ and $\mathcal{L}^{3}$, we get

$$
L_{i}^{[1,3]}:= \begin{cases}L_{0}^{[1,2]} \oplus L_{i}^{3}, & \text { if } 0 \leq i \leq e_{3}^{\prime}-1 \\ L_{i-e_{3}^{\prime}}^{[1,2]} \oplus L_{e_{3}^{\prime}}^{2}, & \text { if } e_{3}^{\prime} \leq i \leq\left(e_{1}^{\prime}+e_{2}^{\prime}\right)+e_{3}^{\prime}-1,\end{cases}
$$

that is, by using (16),

$$
L_{i}^{[1,3]}:= \begin{cases}L_{0}^{1} \oplus L_{2}^{0} \oplus L_{i}^{3}, & \text { if } 0 \leq i \leq e_{3}^{\prime}-1 \\ L_{0}^{1} \oplus L_{i-e_{3}^{\prime}}^{2} \oplus L_{e_{3}^{\prime}}^{2}, & \text { if } e_{3}^{\prime} \leq i \leq e_{2}^{\prime}+e_{3}^{\prime}-1 \\ L_{i-e_{3}^{\prime}-e_{2}^{\prime}}^{1} \oplus L_{e_{2}^{\prime}}^{2} \oplus L_{e_{3}^{\prime}}^{2}, & \text { if } e_{2}^{\prime}+e_{3}^{\prime} \leq i \leq e_{1}^{\prime}+e_{2}^{\prime}+e_{3}^{\prime}-1 .\end{cases}
$$

Using this procedure, we finally obtain an $\mathfrak{o}_{E}$-lattice chain

$$
\mathcal{L}^{1}+\cdots+\mathcal{L}^{m}=\mathcal{L}^{[1, m]}:=\left\{L_{i}^{[1, m]}: i \in \mathbb{Z}\right\}
$$

of period $e_{1}^{\prime}+e_{2}^{\prime}+\cdots+e_{m}^{\prime}$. We have

$$
L_{i}^{[1, m]}=L_{0}^{1} \oplus \cdots \oplus L_{0}^{j-1} \oplus L_{k}^{j} \oplus L_{e_{j+1}^{\prime}}^{j+1} \oplus \cdots \oplus L_{e_{m}^{\prime}}^{m},
$$

for $i=e_{j+1}^{\prime}+\cdots+e_{m}^{\prime}+k$ with $1 \leq j \leq m$ and $0 \leq k \leq e_{j}^{\prime}-1$. 
We will need the $\mathfrak{o}_{E}$-lattice chain $\mathcal{L}^{1}+\cdots+\mathcal{L}^{m}$ in the special case when $e_{1}^{\prime}=\cdots=e_{m}^{\prime}=1$. In that case, the equation (19) becomes

$$
L_{i}^{[1, m]}=L_{0}^{1} \oplus \cdots \oplus L_{0}^{m-i-1} \oplus L_{0}^{m-i} \oplus L_{1}^{m-i+1} \oplus \cdots \oplus L_{1}^{m},
$$

for each $i \in\{0,1, \ldots, m-1\}$.

Since the $\mathfrak{o}_{E}$-lattice chains $\mathcal{L}^{1}, \ldots, \mathcal{L}^{m}$ all have period 1 , we have $\mathfrak{p}_{E}^{j} L_{0}^{l}=$ $L_{j}^{l}$, for each $l \in\{1, \ldots, m\}$ and each $j \in \mathbb{Z}$. Hence, since the $\mathfrak{o}_{E}$-lattice chain $\mathcal{L}^{[1, m]}$ is of period $m$, we have

$$
L_{m j+k}^{[1, m]}=\mathfrak{p}_{E}^{j} L_{k}^{[1, m]}=L_{j}^{1} \oplus \cdots \oplus L_{j}^{m-k-1} \oplus L_{j}^{m-k} \oplus L_{j+1}^{m-k+1} \oplus \cdots \oplus L_{j+1}^{m},
$$

for each $j \in \mathbb{Z}$ and each $k \in\{0,1, \ldots, m-1\}$.

Then we have

$$
L_{m j+k+1}^{[1, m]}=L_{j}^{1} \oplus \cdots \oplus L_{j}^{m-k-1} \oplus L_{j+1}^{m-k} \oplus L_{j+1}^{m-k+1} \oplus \cdots \oplus L_{j+1}^{m} .
$$

It follows that

$$
L_{m j+k}^{[1, m]} / L_{m j+k+1}^{[1, m]} \cong L_{j}^{m-k} / L_{j+1}^{m-k}
$$

for each $j \in \mathbb{Z}$ and each $k \in\{0,1, \ldots, m-1\}$.

Hence, setting $d^{l}:=d\left(\mathcal{L}^{l}\right)$ for any $1 \leq l \leq m$, we obtain

$$
d_{m j+k}\left(\mathcal{L}^{[1, m]}\right)=d_{j}^{m-k}=d_{0}^{m-k}=\operatorname{dim}_{E}\left(V^{m-k}\right)=N_{m-k} /[E: F],
$$

by (10), since $e_{m-k}=1$.

We may consider each $\mathcal{L}^{l}, 1 \leq l \leq m$, as an $\mathfrak{o}_{F}$-lattice chain in the $F$ vector space $V$, of period $e(E \mid F)$ (see $[8,(1.2 .4)]$ ). Then $\mathcal{L}^{1}+\cdots+\mathcal{L}^{m}$, viewed as an $\mathfrak{o}_{F}$-lattice chain, has period $m e(E \mid F)$ (by [8, (1.2.4)]) and the equation (21) shows that it is the same as the chain $\mathcal{L}$ considered in the Example 1.

\subsubsection{Second addition procedure}

We keep assuming $e_{1}^{\prime}=\cdots=e_{m}^{\prime}=1$, and we will now consider the $\mathfrak{o}_{E}$-lattice chain

$$
\mathcal{L}^{m}+\cdots+\mathcal{L}^{1}=\left\{L_{i}^{[m, 1]}: i \in \mathbb{Z}\right\}
$$

We have

$$
L_{m j+k}^{[m, 1]}=L_{j+1}^{1} \oplus \cdots \oplus L_{j+1}^{k} \oplus L_{j}^{k+1} \oplus \cdots \oplus L_{j}^{m},
$$

for each $j \in \mathbb{Z}$ and each $k \in\{0,1, \ldots, m-1\}$.

It gives

$$
L_{m j+k}^{[m, 1]} / L_{m j+k+1}^{[m, 1]} \cong L_{j}^{k+1} / L_{j+1}^{k+1},
$$


for each $j \in \mathbb{Z}$ and each $k \in\{0,1, \ldots, m-1\}$. Hence we obtain

$$
d_{m j+k}\left(\mathcal{L}^{[m, 1]}\right)=d_{j}^{k+1}=d_{0}^{k+1}=\operatorname{dim}_{E}\left(V^{k+1}\right)=N_{k+1} /[E: F],
$$

which in particular does not depend on $m$, in contrast with $d_{m j+k}\left(\mathcal{L}^{[1, m]}\right)$.

As before, we may consider each $\mathcal{L}^{l}, 1 \leq l \leq m$, as an $\mathfrak{o}_{F}$-lattice chain in the $F$-vector space $V$, of period $e(E \mid F)$. Then $\mathcal{L}^{m}+\cdots+\mathcal{L}^{1}$, viewed as an $\mathfrak{o}_{F}$-lattice chain, has period $m e(E \mid F)$.

\section{$5 \quad$ Hereditary $\mathfrak{o}_{F}$-orders}

To any $\mathfrak{o}_{F}$-lattice chain $\mathcal{L}=\left\{L_{i}\right\}$ in $V$ is attached the following sequence of $\mathfrak{o}_{F}$-lattices in $A$

$$
\operatorname{End}_{\mathfrak{o}_{F}}^{n}(\mathcal{L}):=\left\{x \in A: x L_{i} \subset L_{i+n}, i \in \mathbb{Z}\right\},
$$

for each $n \in \mathbb{Z}$. In particular, $\mathfrak{A}=\mathfrak{A}(\mathcal{L}):=\operatorname{End}_{\mathfrak{o}_{F}}^{0}(\mathcal{L})$ is an hereditary $\mathfrak{o}_{F^{-}}$ order in $A$, and $\mathfrak{P}:=\operatorname{End}_{\mathfrak{o}_{F}}^{1}(\mathcal{L})$ is the Jacobson radical of $\mathfrak{A}$. We will set

$$
U(\mathfrak{A}):=\mathfrak{A}^{\times} \quad \text { and } \quad U^{n}(\mathfrak{A}):=1+\mathfrak{P}^{n}, \text { for } n \geq 1
$$

We put

$$
\mathfrak{K}(\mathfrak{A}):=\left\{g \in \operatorname{Aut}_{F}(V): g^{-1} \mathfrak{A} g=\mathfrak{A}\right\}
$$

Definition 3. For any partition $\left(N_{1}, N_{2}, \ldots, N_{r}\right)$ of $N$, we denote by

$$
\mathfrak{A}\left(N_{1}, N_{2}, \ldots, N_{r}\right)
$$

the subset of $\mathrm{M}_{N}(F)$ consisting of the matrices of the following form: the $(i, j)$-block has dimension $N_{i} \times N_{j}, 1 \leq i, j \leq r$, and its entries lie in $\mathfrak{o}_{F}$ if $i \leq j$, in $\mathfrak{p}_{F}$ otherwise. Pictorially,

$$
\mathfrak{A}\left(N_{1}, N_{2}, \ldots, N_{r}\right)=\left(\begin{array}{cccc}
\mathfrak{o}_{F} & \mathfrak{o}_{F} & \cdots & \mathfrak{o}_{F} \\
\mathfrak{p}_{F} & \ddots & \ddots & \vdots \\
\vdots & \ddots & \ddots & \mathfrak{o}_{F} \\
\mathfrak{p}_{F} & \cdots & \mathfrak{p}_{F} & \mathfrak{o}_{F}
\end{array}\right)
$$

Let $e:=e(\mathcal{L})$ and $d_{i}:=d_{i}(\mathcal{L})$. For each $i \in\{0,1, \ldots, e-1\}$, we choose elements $v_{i, h} \in L_{i}, 1 \leq h \leq d_{i}$ such that the cosets $v_{i, h}+L_{i+1}$ form a basis of the $k_{F}$-space $L_{i} / L_{i+1}$. Then

$$
\left(v_{e-1,1}, v_{e-1,2}, \ldots, v_{e-1, d_{e-1}}, v_{e-2,1}, v_{e-2,2}, \ldots, v_{e-2, d_{e-2}}, \ldots, v_{0,1}, v_{0,2}, \ldots, v_{0, d_{0}}\right)
$$


is an $F$-basis of $V$. If we use this basis to identify $A$ with the matrix algebra $\mathrm{M}_{N}(F)$, then $\mathfrak{A}$ becomes identified with $\mathfrak{A}\left(d_{0}, d_{2}, \ldots, d_{e-1}\right)$.

Now let $V^{1}, V^{2}, \ldots, V^{m}$ be $m$ finite-dimensional $F$-vector spaces as in sections 4.1.1, 4.1.2, and let $\mathcal{L}^{1}, \mathcal{L}^{2}, \ldots, \mathcal{L}^{m}$ be $m \mathfrak{o}_{E}$-lattice chains in $V^{1}$, $V^{2}, \ldots, V^{m}$, all of period 1 . We put

$$
\mathfrak{A}^{[m, 1]}:=\mathfrak{A}\left(\mathcal{L}^{[m, 1]}\right),
$$

where $\mathcal{L}^{[m, 1]}$ is defined as in (23]).

Let $\left(m_{1}, \ldots, m_{r}\right)$ be a partition of $m$. For each $i \in\{1, \ldots, r\}$, we set $\underline{m}_{i-1}:=m_{1}+\cdots+m_{i-1}$,

$$
\mathcal{L}^{\left[\underline{m}_{i}, \underline{m}_{i-1}+1\right]}:=\mathcal{L}^{\underline{m}_{i}}+\mathcal{L}^{\underline{m}_{i}-1}+\cdots+\mathcal{L}^{\underline{m}_{i-1}+2}+\mathcal{L}^{\underline{m}_{i-1}+1},
$$

and

$$
\mathfrak{A}^{\left[m_{i}, m_{i-1}+1\right]}:=\mathfrak{A}\left(\mathcal{L}^{\left[\underline{m}_{i}, \underline{m}_{i-1}+1\right]}\right) .
$$

We set $m_{0}:=0$. For each $i \in\{1, \ldots, r\}$, we define $V^{\left[m_{i-1}+1, m_{i}\right]}$ as

$$
V^{\left[m_{i-1}+1, m_{i}\right]}:=V^{\underline{m}_{i-1}+1} \oplus V^{\underline{m}_{i-1}+2} \oplus \cdots \oplus V^{\underline{m}_{i}} .
$$

Let $M\left(m_{1}, \ldots, m_{r}\right)$ denote the stabilizer of the decomposition

$$
V=\bigoplus_{i=1}^{r} V^{\left[m_{i-1}+1, m_{i}\right]}
$$

Lemma 2. We have

$$
M\left(m_{1}, \ldots, m_{r}\right) \cap U\left(\mathfrak{A}^{[m, 1]}\right)=\prod_{i=1}^{r} U\left(\mathfrak{A}^{\left[m_{i}, m_{i-1}\right]}\right) .
$$

Proof. We set $e:=e(E \mid F)$. Let $l \in\{1,2, \ldots, m\}$ and let $j \in\{0,1, \ldots, e-1\}$. Since the $\mathfrak{o}_{E}$-lattice chain $\mathcal{L}^{l}$ has period 1, the equations (9) and (10) give

$$
\operatorname{dim}_{k_{E}} L_{j}^{l} / L_{j+1}^{l}=\operatorname{dim}_{k_{E}} L_{0}^{l} / L_{1}^{l}=\frac{N_{l}}{[E: F]}
$$

It follows that

$$
d_{j}^{l}=\operatorname{dim}_{k_{F}} L_{j}^{l} / L_{j+1}^{l}=\left[k_{E}: k_{F}\right] \operatorname{dim}_{k_{E}} L_{j}^{l} / L_{j+1}^{l}=\left[k_{E}: k_{F}\right] \frac{N_{l}}{[E: F]}=\frac{N_{l}}{e} .
$$

Since $d_{j}^{l}=d_{0}^{l}=N_{l} / e$, we may and do fix an $\mathfrak{o}_{F}$-basis $\mathcal{B}^{l}:=\left(v_{0,1}^{l}, \ldots, v_{0, N_{l}}^{l}\right)$ of $\mathcal{L}^{l}$, chosen to span $L_{0}^{l}$ over $\mathfrak{o}_{F}$. We put

$$
v_{j, h}^{l}:= \begin{cases}v_{0, h}^{l} & \text { if } 1 \leq h \leq \delta_{j}^{l}, \\ \varpi_{F} v_{0, h}^{l} & \text { if } \delta_{j}^{l}+1 \leq h \leq N_{l},\end{cases}
$$


where

$$
\delta_{j}^{l}:=\operatorname{dim}_{k_{F}} L_{j}^{l} / L_{e}^{l}=(e-j) d_{j}^{l}=\frac{e-j}{e} N_{l} .
$$

The $\mathfrak{o}_{F}$-lattice $L_{j}^{l}$ is then the $\mathfrak{o}_{F}$-linear span of the set $\left\{v_{j, 1}^{l}, \ldots, v_{j, N_{l}}^{l}\right\}$, the cosets $v_{j, h}^{l}+L_{j+1}^{l}\left(1 \leq h \leq N_{l} / e\right)$ form a basis of the $k_{F^{-} \text {space }} L_{j}^{l} / L_{j+1}^{l}$, and

$$
\left(v_{e-1,1}^{l}, \ldots, v_{e-1, N_{l} / e}^{l}, \ldots, v_{1,1}^{l}, \ldots, v_{1, N_{l} / e}^{l}, v_{0,1}^{l}, \ldots, v_{0, N_{l} / e}^{l}\right)=\mathcal{B}^{l} .
$$

It follows that, for each $i \in\{1,2, \ldots, r\}$,

$$
\mathcal{B}^{\left[m_{i-1}+1, m_{i}\right]}:=\left(\mathcal{B}^{\underline{m}_{i-1}+1}, \mathcal{B}^{\underline{m}_{i-1}+2}, \ldots, \mathcal{B}^{\underline{m}_{i}}\right)
$$

is an $F$-basis of the vector space $V^{\left[m_{i-1}+1, m_{i}\right]}$ such that the cosets

$$
v_{j, h}^{k+1}+L_{m j+k+1}^{[m, 1]}, \quad \text { for } 1 \leq h \leq N_{k+1} / e,
$$

form a basis of the $k_{F}$-space

$$
L_{m j+k}^{[m, 1]} / L_{m j+k+1}^{[m, 1]} \cong L_{j}^{k+1} / L_{j+1}^{k+1},
$$

by (24).

Let $\mathcal{B}$ denote the $F$-basis of $V$ defined as

$$
\mathcal{B}:=\left(\mathcal{B}^{\left[1, m_{1}\right]}, \mathcal{B}^{\left[m_{1}+1, m_{1}+m_{2}\right]}, \ldots, \mathcal{B}^{\left[m_{r-1}+1, m_{r}\right]}\right) .
$$

We observe that we have by construction

$$
\mathcal{B}=\mathcal{B}^{[1, m]},
$$

where $\mathcal{B}^{[1, m]}$ is the $F$-basis corresponding to the partition $m$.

We will now use the basis $\mathcal{B}$ to identify $A=\operatorname{End}_{F}(V)$ with $\mathrm{M}_{N}(F)$ and use the basis $\mathcal{B}^{\left[\underline{m}_{i-1}+1, \underline{m}_{i}\right]}$ to identify $\operatorname{End}_{F}\left(V^{\left[m_{i-1}+1, m_{i}\right]}\right)$ with $\mathrm{M}_{N(i)}(F)$, where

$$
N(i):=N_{\underline{m}_{i-1}+1}+N_{\underline{m}_{i-1}+2}+\cdots+N_{\underline{m}_{i}} .
$$

Then $\mathfrak{A}^{[m, 1]}$ becomes identified with the matrices of the following form: the $\left(h, h^{\prime}\right)$-block has dimension

$$
d_{h}\left(\mathcal{L}^{[m, 1]}\right) \times d_{h^{\prime}}\left(\mathcal{L}^{[m, 1]}\right), \quad \text { if } 0 \leq h, h^{\prime} \leq m e-1,
$$

and its entries lie in $\mathfrak{o}_{F}$ if $i \leq i^{\prime}$, in $\mathfrak{p}_{F}$ otherwise.

Now the product $\prod_{i=1}^{r} \mathfrak{A}^{\left[\underline{m}_{i}, \underline{m}_{i-1}+1\right]}$ is viewed as diagonally embedded in $\mathrm{M}_{N}(F)$, and $\mathfrak{A}^{\left[\underline{m}_{i}, \underline{m}_{i-1}+1\right]}$ becomes then identified with the matrices of the following form: the $\left(\underline{m}_{i-1} e+j, \underline{m}_{i-1} e+j^{\prime}\right)$-block has dimension

$$
d_{j}\left(\mathcal{L}^{\left[\underline{m}_{i}, \underline{m}_{i-1}+1\right]}\right) \times d_{j^{\prime}}\left(\mathcal{L}^{\left[\underline{m}_{i}, \underline{m}_{i-1}+1\right]}\right), \quad \text { if } 0 \leq j, j^{\prime} \leq m_{i} e-1,
$$

and its entries lie in $\mathfrak{o}_{F}$ if $j \leq j^{\prime}$, in $\mathfrak{p}_{F}$ otherwise. Then the result follows from (25). 


\section{Semisimple types}

Let $G=\mathrm{GL}(N, F)=\mathrm{GL}(V)$ and let $\mathfrak{s}=[M, \sigma]_{G}$ be a point in the Bernstein spectrum $\mathfrak{B}(G)$. The Levi subgroup $M$ is the stabilizer of a decomposition $V=\bigoplus_{l=1}^{m} V^{l}$ of $V$ as a direct sum of nonzero subspaces $V^{l}$. We set $N_{l}:=\operatorname{dim}_{F} V^{l}$, and $A_{l}:=\operatorname{End}_{F}\left(V^{l}\right) \cong \mathrm{M}_{N_{l}}(F)$. Then $N_{1}+\cdots+N_{m}=N$, and $M$ is isomorphic to $\operatorname{GL}\left(N_{1}, F\right) \times \cdots \times \operatorname{GL}\left(N_{m}, F\right)$, and the supercuspidal representation $\sigma$ of $M$ is of the form $\sigma=\pi_{1} \otimes \cdots \otimes \pi_{m}$, where $\pi_{l}$ is an irreducible supercuspidal representation of the group $\operatorname{GL}\left(N_{l}, F\right)$, for $l=1, \ldots, m$. We set $\mathfrak{t}:=[M, \sigma]_{M}$.

By [8, Theorem (8.4.1)], for each $l$, there is a maximal simple type $\left(J^{l}, \lambda^{l}\right)$ occuring in $\pi_{l}$. The pair $\left(J_{M}, \tau_{M}\right):=\left(J^{1} \times \cdots \times J^{m}, \lambda^{1} \otimes \cdots \otimes \lambda^{m}\right)$ is then an $\mathfrak{t}$-type in $M$.

By definition (see [8, (5.5.10)]), for each $l$, there exists an element $\beta_{l} \in A^{l}$ for which the algebra $E_{l}:=F\left[\beta_{l}\right]$ is a field and a principal $\mathfrak{o}_{F}$-order $\mathfrak{A}^{l}$ in $A^{l}$, of period $e\left(E_{l} \mid F\right)$, with Jacobson radical $\mathfrak{P}_{l}$, such that

$$
J^{l}= \begin{cases}J\left(\beta_{l}, \mathfrak{A}^{l}\right) & (\text { as defined in }[8,(3.1 .14)]) \text { if } \beta_{l} \notin F, \\ U\left(\mathfrak{A}^{l}\right) & \text { if } \beta_{l} \in F .\end{cases}
$$

For each $x \in A^{l}$, we will write

$$
\nu_{\mathfrak{A}^{l}}(x):=\max \left\{n \in \mathbb{Z}: x \in \mathfrak{P}_{l}\right\} .
$$

Let $\mathcal{L}^{l}$ denote the $\mathfrak{o}_{E}$-lattice chain defining the maximal $\mathfrak{o}_{E}$-order $\mathfrak{B}^{l}:=$ $\mathfrak{A}^{l} \cap \operatorname{End}_{E}\left(V^{l}\right)$. We have

$$
J\left(\beta, \mathfrak{A}^{l}\right) / J^{1}\left(\beta, \mathfrak{A}^{l}\right)=U\left(\mathfrak{B}^{l}\right) / U^{1}\left(\mathfrak{B}^{l}\right) \cong \mathrm{GL}\left(f_{l}, k_{E}\right) .
$$

\subsection{Simple types}

We assume in this subsection that the $N_{l}$ are all equal to $N / m$ and that $\pi_{l} \cong \pi_{j} \chi_{j}$, with $\chi_{j}$ an unramified character of $\mathrm{GL}(N / m, F)$, for each $l, j \in$ $\{1, \ldots, m\}$. In particular, $M$ is then isomorphic to $\mathrm{GL}(N / m, F)^{m}$, and by 8. Theorem (8.4.2)], we can assume that all the $\beta_{l}$, all the $\mathfrak{A}^{l}$, all the $\mathcal{L}^{l}$, all the $J^{l}$ and all the $\lambda^{l}$ are equal. We will denote by $E$ (resp. $\beta$ ) the common value of the $E_{l}$ (resp. $\beta_{l}$ ).

Using the second addition procedure 4.1.2, we define: the $\mathfrak{o}_{E}$-lattice chain

$$
\mathcal{L}:=\mathcal{L}^{m}+\mathcal{L}^{m-1}+\cdots+\mathcal{L}^{1}, \quad \text { and } \quad \mathfrak{A}:=\operatorname{End}_{\mathfrak{o}_{F}}^{0}(\mathcal{L}) .
$$

If $\beta$ belongs to $F$, we set $J:=U(\mathfrak{A})$. Otherwise, let $n:=-\nu_{\mathfrak{A}^{1}}(\beta)$, then $[\mathfrak{A}, m n, 0, \beta]$ is a simple stratum in the sense of [8, Definition (1.5.5)], let $(J, \lambda):=(J(\beta, \mathfrak{A}), \lambda)$ be the corresponding simple type in $G$. 
Let $\mathfrak{B}$ denote the principal $\mathfrak{o}_{E}$-order in $B:=\mathrm{M}_{N /[E: F]}(E)$ defined by $\mathfrak{B}:=B \cap \mathfrak{A}$. We have $m=e(\mathfrak{B})=e\left(\mathfrak{B} \mid \mathfrak{o}_{E}\right)$. In the case when $\beta \in F$, we have $m=e(\mathfrak{A})$.

Definition 4. We set

$$
\mathfrak{A}^{\mathfrak{s}}:=\mathfrak{A}(N / m, \ldots, N / m) \quad \text { and } \quad J^{\mathfrak{s}}:=U\left(\mathfrak{A}^{\mathfrak{s}}\right),
$$

where $\mathfrak{A}(N / m, \ldots, N / m)$ is defined by Definition [3.

Lemma 3. The $\mathfrak{o}_{F}$-order $\mathfrak{A}$ is contained in the $\mathfrak{o}_{F}$-order $\mathfrak{A}^{\mathfrak{F}}$.

Proof. We have $\mathfrak{A}=\mathfrak{A}(N / e(\mathfrak{A}), \ldots, N / e(\mathfrak{A}))$. In the case when $J=U(\mathfrak{A})$, we have $\mathfrak{A}^{\mathfrak{s}}=\mathfrak{A}$. Otherwise, the statement follows immediately from the above descriptions of the orders $\mathfrak{A}, \mathfrak{A}^{\mathfrak{s}}$, and from the fact (see [8, Proposition (1.2.4)]) that

$$
e(\mathfrak{A})=m \cdot e(E \mid F) .
$$

Indeed, from the above descriptions of the orders $\mathfrak{A}, \mathfrak{A}^{\mathfrak{s}}$, we have

$$
\begin{gathered}
\mathfrak{A}^{\mathfrak{s}} \cap U=\mathfrak{A} \cap U, \mathfrak{A}^{\mathfrak{s}} \cap \bar{U}=\mathfrak{A} \cap \bar{U}, \\
M \cap \mathfrak{A}^{\mathfrak{s}} \cong\left(\mathrm{GL}\left(N / m, \mathfrak{o}_{F}\right)\right)^{m},
\end{gathered}
$$

while $M \cap \mathfrak{A}$ is isomorphic to the product of $m$ copies of the order of $e(E \mid F) \times$ $e(E \mid F)$ blocks matrices of the following form: the $(j, l)$-block has dimension $N / e(\mathfrak{A}) \times N / e(\mathfrak{A})=(N / e(E \mid F) m \times N / e(E \mid F) m), 0 \leq j, l \leq e(E \mid F)-1$, and its entries lie in $\mathfrak{o}_{F}$ if $j \leq l$, in $\varpi_{F} \mathfrak{o}_{F}$ otherwise, so that $M \cap \mathfrak{A} \subset M \cap \mathfrak{A}^{\mathfrak{s}}$.

We set

$$
f=\frac{N}{[E: F] \cdot m} .
$$

Let $K / E$ be an unramified field extension of degree $f$ with

$$
K^{\times} \subset \mathfrak{K}(\widetilde{\mathfrak{B}}),
$$

where $\mathfrak{K}(\widetilde{\mathfrak{B}})$ is defined by (27), and let $C=\operatorname{End}_{K}(V) \cong \mathrm{M}_{m}(K)$. We view $\varpi_{E}$ as a prime element of $K$. For $i=1, \ldots, m-1$, let $s_{i, C}$ denote the matrix in $C$ of the transposition $i \leftrightarrow i+1$, that is,

$$
s_{i, C}=\left(\begin{array}{cccc}
\mathrm{I}_{i-1} & & & \\
& 0 & 1 & \\
& 1 & 0 & \\
& & & \mathrm{I}_{m-i-1}
\end{array}\right),
$$


and let $s_{0, C}=\Pi_{m, C} s_{1, C} \Pi_{m, C}^{-1}$, with

$$
\Pi_{m, C}=\left(\begin{array}{cc}
0 & \mathrm{I}_{m-1} \\
\varpi_{E} & 0
\end{array}\right)
$$

We fix the embedding

$$
\bigotimes \mathrm{I}_{N / m}: C \hookrightarrow \mathrm{M}_{N}(K) \quad c=\left(c_{i j}\right) \mapsto c \otimes \mathrm{I}_{N / m}=\left(c_{i j} \mathrm{I}_{N / m}\right),
$$

$c \otimes \mathrm{I}_{N / m}$ being a block matrix with scalar blocks.

Let $\widetilde{W}_{C}$ be the group generated by

$$
S=\left\{s_{0, C} \otimes \mathrm{I}_{N / m}, s_{1, C} \otimes \mathrm{I}_{N / m}, \ldots, s_{m-1, C} \otimes \mathrm{I}_{N / m}\right\}
$$

Then $\left(\widetilde{W}_{C}, S\right)$ is a Coxeter group of type $\tilde{A}_{m-1}$.

Theorem 4. The representation $\alpha=\operatorname{Ind}_{J}^{J^{\mathfrak{s}}}(\lambda)$ is irreducible. Hence the pair $\left(J^{\mathfrak{s}}, \alpha\right)$ is an $\mathfrak{s}$-type.

Proof. In the case when $J=U(\mathfrak{A})$, we have $J^{\mathfrak{s}}=J$, so the result follows trivially in this case. We will assume from now on that $J=J(\beta, \mathfrak{A})$. For any $i \in\{1, \ldots, m-1\}$,

$$
s_{i, C} \otimes \mathrm{I}_{N / m}=\left(\begin{array}{cccc}
\mathrm{I}_{(i-1) N / m} & & & \\
& 0 & \mathrm{I}_{N / m} & \\
& \mathrm{I}_{N / m} & 0 & \\
& & & \mathrm{I}_{(m-i-1) N / m}
\end{array}\right) \notin J^{\mathfrak{s}}
$$

and

$$
\left.\Pi_{m, C} \otimes \mathrm{I}_{N / m}=\left(\begin{array}{cc}
0 & \mathrm{I}_{(m-1) N / m} \\
\varpi_{E} \mathrm{I}_{N / m} & 0
\end{array}\right) \notin J^{\mathfrak{s}}\right) .
$$

Hence $\widetilde{W}_{C} \cap J^{\mathfrak{s}}=\{1\}$, which gives

$$
J^{\mathfrak{s}} \cap\left(J \cdot \widetilde{W}_{C} \cdot J\right)=J
$$

Then the result follows from the fact (see [8, Propositions (5.5.11) and (5.5.14) (iii)]) that

$$
I_{G}(\lambda) \subset J \cdot \widetilde{W}_{C} \cdot J
$$




\subsection{In the Levi subgroup $\widetilde{M}$}

We will now consider the case of an arbitrary point $\mathfrak{s}=[M, \sigma]_{G}$ in $\mathfrak{B}(G)$, with $G=\operatorname{GL}(N, F)$. Let $\widetilde{M}$ denote the unique Levi subgroup of $G$ which contains $\mathrm{N}_{\mathrm{t}}$ (see 四) and is minimal for this property.

We write $\sigma=\pi_{1} \otimes \pi_{2} \otimes \cdots \otimes \pi_{m}$ as

$$
\sigma=\left(\sigma_{1}, \ldots, \sigma_{1}, \sigma_{2}, \ldots, \sigma_{2}, \ldots, \sigma_{t}, \ldots, \sigma_{t}\right),
$$

where $\sigma_{j}$, a supercuspidal representation of $\operatorname{GL}\left(N_{j}^{\prime}, F\right)$, is repeated $\varepsilon_{j}$ times, $1 \leq j \leq t$, and $\sigma_{1}, \ldots, \sigma_{t}$ are pairwise distinct (after unramified twist). The integers $\varepsilon_{1}, \varepsilon_{2}, \ldots, \varepsilon_{t}$ are called the exponents of $\sigma$. Then we have

$$
M \cong \mathrm{GL}\left(N_{1}^{\prime}, F\right)^{\varepsilon_{1}} \times \mathrm{GL}\left(N_{2}^{\prime}, F\right)^{\varepsilon_{2}} \times \cdots \times \mathrm{GL}\left(N_{t}^{\prime}, F\right)^{\varepsilon_{t}},
$$

and

$$
\widetilde{M} \cong \mathrm{GL}\left(\varepsilon_{1} N_{1}^{\prime}, F\right) \times \mathrm{GL}\left(\varepsilon_{2} N_{2}^{\prime}, F\right) \times \cdots \times \mathrm{GL}\left(\varepsilon_{t} N_{t}^{\prime}, F\right) .
$$

For every $j \in\{1, \ldots, t\}$, we set

$$
\mathfrak{s}_{j}=\left[\mathrm{GL}\left(N_{j}^{\prime}, F\right)^{\varepsilon_{j}}, \sigma_{j}^{\otimes \varepsilon_{j}}\right]_{\mathrm{GL}\left(\varepsilon_{j} N_{j}^{\prime}, F\right)} .
$$

Then let $\left(K^{j}, \tau^{j}\right)$ be the $\mathfrak{s}_{j}$-type in $\mathrm{GL}\left(\varepsilon_{j} N_{j}^{\prime}, F\right)$ (a simple type) defined as in the previous section, and let $\left(\widetilde{K}^{j}, \widetilde{\tau}^{j}\right)$ be the "modified simple type" attached to $\left(K^{j}, \tau^{j}\right)$ as in [10, proof of Prop. 1.4].

Lemma 4. We have $\widetilde{K}^{j} \subset J^{\mathfrak{s}_{j}}$ and $\alpha_{i}=\operatorname{Ind}_{\widetilde{K}^{j}}^{J^{\mathfrak{s}_{j}}}\left(\widetilde{\tau}^{j}\right)$ is irreducible.

Proof. There is an isomorphism of Hecke algebras

$$
\mathcal{H}\left(\mathrm{GL}\left(\varepsilon_{j} N_{j}^{\prime}\right), \widetilde{\tau}^{j}\right) \cong \mathcal{H}\left(\mathrm{GL}\left(\varepsilon_{j} N_{j}\right), \tau^{j}\right)
$$

such that, if $\tilde{f} \in \mathcal{H}\left(\operatorname{GL}\left(\varepsilon_{j} N_{j}^{\prime}\right), \widetilde{\tau}^{j}\right)$ has support $\widetilde{K}^{j} g \widetilde{K}^{j}$, for some element $g \in \mathrm{GL}\left(\varepsilon_{j} N_{j}^{\prime}, F\right)$, then its image $f$ in $\mathcal{H}\left(\mathrm{GL}\left(\varepsilon_{j} N_{j}^{\prime}\right), \tau^{j}\right)$ has support $K^{j} g K^{j}$ (see [8, (7.2.19)]). Then the result follows from Theorem 4 .

We set

$$
\begin{gathered}
\mathfrak{s}_{\widetilde{M}}=[M, \sigma]_{\widetilde{M}}, \quad \widetilde{\mathfrak{A}}^{\mathfrak{s}}=\mathfrak{A}^{\mathfrak{s}_{1}} \times \cdots \times \mathfrak{A}^{\mathfrak{s}_{t}}, \quad \widetilde{J}^{\mathfrak{s}}=U\left(\widetilde{\mathfrak{A}}^{\mathfrak{s}}\right), \\
\widetilde{K}=\widetilde{K}^{1} \times \cdots \times \widetilde{K}^{t} \subset \widetilde{M}, \quad \widetilde{\tau}=\widetilde{\tau}^{1} \otimes \cdots \otimes \widetilde{\tau}^{t} .
\end{gathered}
$$

Note that

$$
\widetilde{J}^{\mathfrak{s}}=\widetilde{M} \cap J^{\mathfrak{s}} .
$$

It immediately follows from Lemma 4 that:

Lemma 5. We have $\widetilde{K} \subset \widetilde{J}^{\mathfrak{s}}$ and $\widetilde{\alpha}=\operatorname{Ind}_{\widetilde{K}}^{\widetilde{J}^{s}}(\widetilde{\tau})$ is irreducible. 


\subsection{Review of endo-classes}

We recall that a simple pair $(k, \beta)$ over $F$ consists of an integer $k$ and a nonzero element $\beta$ generating a field extension $E$ of $F$ such that

$$
-k>\max \left\{k_{0}(\beta, \mathfrak{A}(E)), \nu_{E}(\beta)\right\},
$$

where $\nu_{E}$ is the standard additive valuation on $E$ and $k_{0}(\beta, \mathfrak{A}(E))$ is defined by [8. (1.4.5)], with $\mathfrak{A}(E)$ denoting the unique hereditary $\mathfrak{o}_{F}$-order in $\operatorname{End}_{F}(E)$ such that $\mathfrak{K}(\mathfrak{A}(E)) \supset E^{\times}$.

Let $(k, \beta)$ be a given simple pair in which $k \geq 0$. A ps-character (attached to the simple pair $(k, \beta))$ is then a triple $(\Theta, k, \beta)$, where $\Theta$ is a simplecharacter-valued function, such that to each triple $(V, \mathfrak{B}, m)$, where $V$ is a finite-dimensional $E$-vector space, $\mathfrak{B}$ is a hereditary $\mathfrak{o}_{E}$-order in $\operatorname{End}_{E}(V)$, and $m$ is an integer such that $\left[m / e\left(\mathfrak{B} \mid \mathfrak{o}_{E}\right)\right]=k$, the function $\Theta$ attaches a simple character $\Theta(\mathfrak{A}) \in \mathcal{C}(\mathfrak{A}, m, \beta)$, called the realization of $\Theta$ on $\mathfrak{A}$ of order $m$. (If we put $n:=-\nu_{E}(\beta) e(\mathfrak{B})$, the stratum $[\mathfrak{A}, n, m, \beta]$ is simple and the simple character set $\mathcal{C}(\mathfrak{A}, m, \beta)$ of $[\mathbb{8}$, (3.2)] is defined.)

These realizations are subject to the following coherence condition: if we have two realizations $\Theta\left(\mathfrak{A}_{1}\right)$ and $\Theta\left(\mathfrak{A}_{2}\right)$ of on orders $\mathfrak{A}_{1}, \mathfrak{A}_{2}$, they are related by $\Theta\left(\mathfrak{A}_{2}\right)=\tau_{\mathfrak{A}_{1}, \mathfrak{A}_{2}, \beta}\left(\Theta\left(\mathfrak{A}_{1}\right)\right)$, where

$$
\tau_{\mathfrak{A}_{1}, \mathfrak{A}_{2}, \beta}: \mathcal{C}\left(\mathfrak{A}_{1}, m, \beta\right) \rightarrow \mathcal{C}\left(\mathfrak{A}_{2}, m, \beta\right)
$$

is the canonical bijection of $[8,(3.6 .14)]$.

Following [10, §4.3], we will say that two ps-characters $\left(\Theta_{1}, k_{1}, \beta_{1}\right)$ and $\left(\Theta_{2}, k_{2}, \beta_{2}\right)$ are endo-equivalent if there exists an $F$-vector space $V$, hereditary $\mathfrak{o}_{F}$-orders $\mathfrak{A}_{1}, \mathfrak{A}_{2}$ in $\operatorname{End}_{F}(V)$, and realizations $\Theta_{i}\left(\mathfrak{A}_{i}\right)$ of the $\Theta_{i}$ of same level, such that $\mathfrak{A}_{1} \cong \mathfrak{A}_{2}$ as $\mathfrak{o}_{F}$-orders, and such that the simple characters $\Theta_{i}\left(\mathfrak{A}_{i}\right)$

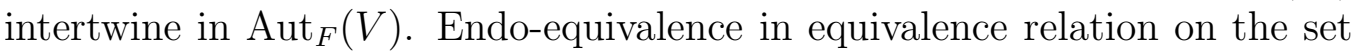
of ps-characters over $F$. One refers to the equivalence classes as endo-classes of simple characters.

If the supercuspidal representation $\pi_{l}$ of $\mathrm{GL}\left(N_{l}, F\right)$ contains the trivial character of $U^{1}\left(\mathfrak{A}^{l}\right)=1+\mathfrak{P}_{l}$, then $\pi_{l}$ is said to be of level-zero. Otherwise, there exists a simple stratum $\left[\mathfrak{A}^{l}, n_{l}, 0, \beta_{l}\right]$ in $A_{l}$ and a simple character $\theta_{l} \in \mathcal{C}\left(\mathfrak{A}^{l}, 0, \beta_{l}\right)$ such that the restriction of $\lambda^{l}$ to $H^{1}\left(\beta_{l}, \mathfrak{A}^{l}\right)$ is a multiple of $\theta_{l}$. (Here $H^{1}\left(\beta_{l}, \mathfrak{A}^{l}\right)$ is defined as in [8, (3.1.14)].) Since $\left[\mathfrak{A}^{l}, n_{l}, 0, \beta_{l}\right]$ is simple, we have $n_{l}=-\nu_{\mathfrak{A l}^{l}}\left(\beta_{l}\right)$. Then each representation $\lambda^{l}$ is given as follows. There is a unique irreducible representation $\eta_{l}$ of $J^{1}\left(\beta, \mathfrak{A}^{l}\right)$ whose restriction to $H^{1}\left(\beta, \mathfrak{A}^{l}\right)$ is a multiple of $\theta_{l}$. The representation $\eta_{l}$ extends to a representation $\kappa_{l}$ which is a $\beta$-extension of $\eta_{l}$, and we have $\lambda^{l}=\kappa_{l} \otimes \rho_{l}$, where $\rho_{l}$ is the inflation of an irreducible representation of $\operatorname{GL}\left(f_{l}, k_{E}\right)$, with $f_{l}$ defined by (30). 
If the representation $\pi_{l}$ is of level zero, we set $\Theta_{\pi_{l}}=\left\{\Theta^{0}\right\}$, where $\Theta^{0}$ is the trivial ps-character (that is, if $\mathfrak{A}$ is a hereditary $\mathfrak{o}_{F}$-order in some $\operatorname{End}_{F}(V)$, the realization of $\Theta^{0}$ on $\mathfrak{A}$ is the trivial character of $U^{1}(\mathfrak{A})$ ). Otherwise, the simple character $\theta_{i}$ determines a ps-character $\left(\Theta_{l}, 0, \beta\right)$ and hence an endoclass $\Theta_{\pi_{l}}$.

We will denote by $\Theta(1), \Theta(2), \ldots, \Theta(q)$ the distinct endo-classes arising in the set $\left\{\Theta_{\pi_{1}}, \ldots, \Theta_{\pi_{m}}\right\}$.

\subsection{The homogeneous case}

In this subsection, we assume that all the representations $\pi_{1}, \pi_{2}, \ldots, \pi_{m}$ admit the same endo-class. It follows that all the elements $\beta_{1}, \ldots, \beta_{m}$ may be assumed to be equal. We will denote by $E$ (resp. $\beta$ ) the common value of the $E_{l}$ (resp. $\beta_{l}$ ).

Let $l \in\{1, \ldots, m\}$, and let $\left(v_{1}^{l}, v_{2}^{l}, \ldots, v_{N_{l}}^{l}\right)$ be an $F$-basis of $V^{l}$, with respect to which $\mathfrak{A}^{l}=\mathfrak{A}\left(\mathcal{L}^{l}\right)$ is identified with $\mathfrak{A}\left(N_{l} / e(E \mid F), \ldots, N_{l} / e(E \mid F)\right)$. We have $L_{0}^{l}=\mathfrak{o}_{F} v_{1}^{l} \oplus \cdots \oplus \mathfrak{o}_{F} v_{N_{l}}^{l}$. We set

$$
L_{i, \max }^{l}:=\mathfrak{p}^{i} L_{0}^{l}, \text { for any } i \in \mathbb{Z} \text {. }
$$

Then

$$
\mathcal{L}_{\text {max }}^{l}:=\left\{L_{i, \max }^{l}: i \in \mathbb{Z}\right\}
$$

is an $\mathfrak{o}_{F}$-lattice chain in $V^{l}$ of period 1 , and we have

$$
\mathfrak{A}\left(\mathcal{L}_{\max }^{l}\right):=\operatorname{End}_{\mathfrak{o}_{F}}^{0}\left(\mathcal{L}_{\max }^{l}\right)=\mathfrak{A}\left(N_{l}\right)=\mathrm{M}_{N_{l}}\left(\mathfrak{o}_{F}\right) \supset \mathfrak{A}^{l}
$$

Following the second addition procedure defined in the subsection 4.1, we assemble the $\mathfrak{o}_{E}$-lattices chains $\mathcal{L}^{1}, \ldots, \mathcal{L}^{m}$ into the $\mathfrak{o}_{E}$-lattice chain

$$
\overline{\mathcal{L}}:=\mathcal{L}^{m}+\mathcal{L}^{m-1}+\cdots+\mathcal{L}^{1}
$$

in $V$, of period $m$, and we assemble the $\mathfrak{o}_{F}$-lattices chains $\mathcal{L}_{\max }^{1}, \ldots, \mathcal{L}_{\max }^{m}$ into the $\mathfrak{o}_{F}$-lattice chain

$$
\overline{\mathcal{L}}_{\text {max }}:=\mathcal{L}_{\max }^{m}+\mathcal{L}_{\max }^{m-1}+\cdots+\mathcal{L}_{\text {max }}^{1}=\left\{\bar{L}_{\max , i}: i \in \mathbb{Z}\right\}
$$

in $V$, of period $m$. Let $j \in \mathbb{Z}$ and $k \in\{0,1, \ldots, m-1\}$. From (24), we have

$$
\bar{L}_{\max , m j+k} / \bar{L}_{\max , m j+k+1} \cong L_{\max , j}^{k+1} / L_{\max , j+1}^{k+1} .
$$

Hence:

$$
d_{m j+k}\left(\overline{\mathcal{L}}_{\text {max }}\right)=N_{k+1}
$$


It follows that

$$
\mathfrak{A}\left(\overline{\mathcal{L}}_{\text {max }}\right)=\mathfrak{A}\left(N_{1}, N_{2}, \ldots, N_{m}\right)
$$

We put

$$
B:=\operatorname{End}_{E}(V) \text { and } \mathfrak{B}:=\operatorname{End}_{\mathfrak{o}_{E}}^{0}(\overline{\mathcal{L}}) .
$$

Considering $\overline{\mathcal{L}}$ as an $\mathfrak{o}_{F}$-lattice chain, we put

$$
\mathfrak{A}:=\operatorname{End}_{\mathfrak{o}_{F}}^{0}(\overline{\mathcal{L}}) .
$$

We have $\mathfrak{B}=\mathfrak{A} \cap B$.

The following definition, lemma and theorem generalize Definition 4, Lemma 3, and Theorem 4, respectively.

Definition 5. We set

$$
\mathfrak{A}^{\mathfrak{s}}:=\mathfrak{A}\left(N_{1}, N_{2}, \ldots, N_{m}\right) \quad \text { and } \quad J^{\mathfrak{s}}:=U\left(\mathfrak{A}^{\mathfrak{s}}\right)
$$

Lemma 6. The $\mathfrak{o}_{F}$-order $\mathfrak{A}$ is contained in the $\mathfrak{o}_{F}$-order $\mathfrak{A}^{\mathfrak{s}}$.

Proof. We have

$$
\begin{gathered}
\mathfrak{A}^{\mathfrak{s}} \cap U=\mathfrak{A} \cap U, \quad \mathfrak{A}^{\mathfrak{s}} \cap \bar{U}=\mathfrak{A} \cap \bar{U}, \\
M \cap \mathfrak{A}^{\mathfrak{s}} \cong \prod_{l=1}^{m} \operatorname{GL}\left(N_{l}, \mathfrak{o}_{F}\right) .
\end{gathered}
$$

In the notation of subsection 4.1.2, setting $\left(m_{1}, \ldots, m_{r}\right)=(1, \ldots, 1)$, we have $r=m, M=M\left(m_{1}, \cdots, m_{r}\right)$. Then $\underline{m}_{l-1}=l-1=\underline{m}_{l}-1$, hence $\overline{\mathcal{L}}^{\left[\underline{m}_{l}, \underline{m}_{l-1}+1\right]}=\mathcal{L}^{l}$,

$$
\mathfrak{A}^{l}=\mathfrak{A}\left(\mathcal{L}^{l}\right) \cong \mathfrak{A}\left(N_{l} / e(E \mid F), \ldots, N_{l} / e(E \mid F)\right),
$$

and Lemma 2 gives

$$
M \cap \mathfrak{A} \cong \prod_{l=1}^{m} U\left(\mathfrak{A}^{l}\right) .
$$

Since $U\left(\mathfrak{A}^{l}\right) \subset \operatorname{GL}\left(N_{l}, \mathfrak{o}_{F}\right)$, the result follows.

We set

$$
n:=\max \left(n_{1}, \ldots, n_{m}\right) .
$$

Lemma 7. With notation as above, $[\mathfrak{A}, n m, 0, \beta]$ is a simple stratum.

Proof. We have to check that the four conditions occuring in 8, Definition (1.5.5)] are satisfied. 
(i) We know that the algebra $E=F[\beta]$ is a field, since the strata $\left[\mathfrak{A}^{l}, n_{l}, 0, \beta\right]$ are simple.

(ii) We defined $\overline{\mathcal{L}}=\left\{\bar{L}_{i}: i \in \mathbb{Z}\right\}$ to be an $\mathfrak{o}_{E}$-lattice chain in the $E$-vector space $V$. Hence, by [8, Proposition (1.2.1)], we have $E^{\times} \subset \mathfrak{K}(\mathfrak{A})$.

(iii) Let $l \in\{1, \ldots, m\}$. We set $\mathfrak{Q}_{l}:=\mathfrak{B}_{l} \cap \mathfrak{P}_{l}$. Since $\nu_{\mathfrak{A}^{l}}(\beta)=-n_{l}$, the definition (29) for $\nu_{\mathfrak{A}^{l}}$ shows that

$$
\beta \in \operatorname{End}_{E}\left(V^{l}\right) \cap \mathfrak{P}_{l}^{-n_{l}} \text { and } \beta \notin \mathfrak{P}_{l}^{-n_{l}+1},
$$

that is,

$$
\beta \in \mathfrak{Q}_{l}^{-n_{l}} \text { and } \beta \notin \mathfrak{Q}_{l}^{-n_{l}+1} .
$$

By [8, Proposition (1.2.4)], we know that $\mathfrak{Q}_{l}$ is the Jacobson radical of the $\mathfrak{o}_{E}$-order $\mathfrak{B}_{l}$. Hence $\mathfrak{Q}_{l}^{i}=\operatorname{End}_{\mathfrak{o}_{E}}^{i}\left(\mathcal{L}^{l}\right)$ for each $i \in \mathbb{Z}$, and $\beta\left(L_{j}^{l}\right)$ is contained in $L_{j-n_{l}}^{l}$ and not in $L_{j-n_{l}+1}^{l}$. Now, it follows from (23) that

$$
\beta\left(L_{m j+k}\right)=\beta\left(L_{j+1}^{1}\right) \oplus \cdots \oplus \beta\left(L_{j+1}^{k}\right) \oplus \beta\left(L_{j}^{k+1}\right) \oplus \cdots \oplus \beta\left(L_{j}^{m}\right),
$$

for each $j \in \mathbb{Z}$ and each $k \in\{0,1, \ldots, m-1\}$. Since $n=\max \left(n_{1}, \ldots, n_{m}\right)$, we have $L_{j-n_{l}}^{l} \subset L_{j-n}^{l}$, for each $l$. It gives $\beta\left(L_{m j+k}\right) \subset L_{m(j-n)+k}$. On the other side there exists $l_{0} \in\{1, \ldots, m\}$ such that $n=n_{l_{0}}$, and hence $\beta\left(L_{j}^{l_{0}}\right)$ is not contained in $L_{j-n+1}^{l}$. It follows that $\beta\left(L_{m j+k}\right)$ is not contained in $L_{m(j-n)+k+1}$, that is,

$$
\beta \in \mathfrak{Q}^{-n} \text { and } \beta \notin \mathfrak{Q}^{-n+1},
$$

where $\mathfrak{Q}$ denotes the Jacobson radical of $\mathfrak{B}$. Since $\mathfrak{Q}^{i}=B \cap \mathfrak{P}^{i}$ for each $i \in \mathbb{Z}$ (by [8, Proposition (1.2.4)]), we get $\nu_{\mathfrak{A}}(\beta)=-n m$.

(iv) Let $A(E):=\operatorname{End}_{F}(E)$. The algebra $A(E)$ contains the principal $\mathfrak{o}_{F^{-}}$ order

$$
\mathfrak{A}(E):=\operatorname{End}_{\mathfrak{o}_{F}}^{0}\left(\left\{\mathfrak{p}_{E}^{i}: i \in \mathbb{Z}\right\}\right) .
$$

We have $E^{\times} \subset \mathfrak{K}(\mathfrak{A}(E))$ and [8, Proposition (1.4.13) (ii)] gives

$$
k_{0}(\beta, \mathfrak{A})=m k_{0}(\beta, \mathfrak{A}(E))=k_{0}\left(\beta, \mathfrak{A}^{l}\right), \quad \text { for each } l \in\{1, \ldots, m\} .
$$

Since $\left[\mathfrak{A}^{l}, n_{l}, 0, \beta\right]$ is a simple stratum, we have

$$
0<-k_{0}\left(\beta, \mathfrak{A}^{l}\right) .
$$

Hence $0<-k_{0}(\beta, \mathfrak{A})$ and $[\mathfrak{A}, m n, 0, \beta]$ is simple. 
Since $[\mathfrak{A}, n m, 0, \beta]$ is a simple stratum, we can associate to it the compact open subgroups $J(\beta, \mathfrak{A})$ and $H^{1}(\beta, \mathfrak{A})$ of $U(\mathfrak{A})$, defined following [으, (3.1.14)].

As in [8, $§ 7.1,7.2]$, the set

$$
K:=H^{1}(\beta, \mathfrak{A}) \cap \bar{U} \cdot J(\beta, \mathfrak{A}) \cap P
$$

is then a subgroup of $U(\mathfrak{A})$ containing $H^{1}(\beta, \mathfrak{A})$.

Definition [5] and Lemma [6] imply

$$
K \subset J^{\mathfrak{s}}
$$

As in 10, $\$ 7.2 .1$ ], it admits an irreducible representation $\kappa$, trivial on $K \cap \bar{U}$, $K \cap U$, whose restriction to $H^{1}(\beta, \mathfrak{A})$ is a multiple of $\theta=\Theta(\mathfrak{A})$, and such that $\kappa_{\mid K \cap M}$ is of the form $\kappa_{1}^{\prime} \otimes \cdots \otimes \kappa_{m}^{\prime}$ for some $\beta$-extension $\kappa_{l}^{\prime}$ of $\eta_{l}$. As in [10, $\$ 7.2]$, we can choose the decomposition $\lambda^{l}=\kappa_{l} \otimes \rho_{l}$ above so that $\kappa_{l}=\kappa_{l}^{\prime}$; we assume this has been done. We have canonically

$$
K / K \cap J^{1}(\beta, \mathfrak{A}) \cong \prod_{l=1}^{m} J\left(\beta, \mathfrak{A}^{l}\right) / J^{1}\left(\beta, \mathfrak{A}^{l}\right) \cong \prod_{l=1}^{m} \mathrm{GL}\left(f_{l}, k_{E}\right),
$$

and we can inflate the cuspidal representation $\rho_{1} \otimes \cdots \otimes \rho_{m}$ of $\prod_{l=1}^{m} \operatorname{GL}\left(f_{l}, k_{E}\right)$ to a representation $\rho$ of $K$ and form

$$
\tau=\kappa \otimes \rho
$$

Moreover similar proofs of those of [10, Theorem 7.2.1, Main Theorem 8.2] show that $(K, \tau)$ is a $G$-cover of the pair $(\widetilde{K}, \widetilde{\tau})$ defined in (36) and give the following formula for the intertwining:

$$
\mathcal{I}_{G}(\tau)=K \cdot \mathcal{I}_{\widetilde{M}}(\widetilde{\tau}) \cdot K
$$

Theorem 5. Let $J^{\mathfrak{s}}$ be as in Definition 5. Then the representation $\alpha:=$ $\operatorname{Ind}_{K}^{J^{\mathfrak{s}}}(\tau)$ is irreducible. Hence the pair $\left(J^{\mathfrak{s}}, \alpha\right)$ is an $\mathfrak{s}$-type.

Proof. Using equations (46) and (48), we obtain

$$
\mathcal{I}_{J^{\mathfrak{s}}}(\tau)=K \cdot \mathcal{I}_{\widetilde{M} \cap J^{\mathfrak{s}}}(\widetilde{\tau}) \cdot K .
$$

On the other side, equation (37) and Lemma 5 imply that

$$
\mathcal{I}_{\widetilde{M} \cap J^{\mathfrak{s}}}(\widetilde{\tau})=\widetilde{K} \subset K
$$

Hence $\mathcal{I}_{J^{\mathfrak{s}}}(\tau)=K$, and the result follows from Proposition 2 . 


\subsection{The general case}

The Levi subgroup $\widetilde{M}$ defined in the beginning of the subsection 6.2 is the $G$-stabilizer of a decomposition

$$
V=\widetilde{V}^{1} \oplus \widetilde{V}^{2} \oplus+\cdots \oplus \widetilde{V}^{t},
$$

of $V$ as a direct sum of nonzero subspaces $\widetilde{V}^{j}$.

Since the endo-class of a supercuspidal representation only depends on the corresponding point in the Bernstein spectrum (see [10, Proposition 4.5]), we can associate to each $\widetilde{V}^{j}$ an endo-class of simple characters, namely $\Theta_{\pi_{l}}$ for any $l$ such that $V^{l} \subset \widetilde{V}^{j}$.

Now let $\bar{M} \supset M$ be the Levi subgroup in $G$ defined as in [10, §8.1], that is, for each $i$, let $\bar{V}^{i}$ be the sum of those $\widetilde{V}^{j}$ whose associate endo-class $\Theta_{\pi_{j}}$ is $\Theta(i)$, and write $\bar{M}$ for the $G$-stabilizer of a decomposition

$$
V=\bar{V}^{1} \oplus \bar{V}^{2} \oplus \cdots \oplus \bar{V}^{q}
$$

Setting $\bar{N}_{i}:=\operatorname{dim}_{F} \bar{V}^{i}$, we get

$$
\bar{M} \cong \mathrm{GL}\left(\bar{N}_{1}, F\right) \times \cdots \times \mathrm{GL}\left(\bar{N}_{q}, F\right) .
$$

We put

$$
\bar{K}:=K_{1} \times K_{2} \times \cdots \times K_{q} \text { and } \bar{\tau}:=\tau_{1} \times \tau_{2} \times \cdots \times \tau_{q},
$$

where the pairs $\left(K_{i}, \tau_{i}\right)$ are defined as in (45), (47). Then a similar proof as those of [10, $\S 7.2]$ shows that the pair $(\bar{K}, \bar{\tau})$ is a $\bar{M}$-cover of $\left(J_{M}, \tau_{M}\right)$.

For each $i \in\{1, \ldots, q\}$, let $\overline{\mathcal{L}}^{i}, \overline{\mathcal{L}}_{\text {max }}^{i}$ respectively denote the $\mathfrak{o}_{E}$-lattice chain in the $E$-vector space $\bar{V}^{i}$ defined by (39), and the $\mathfrak{o}_{F}$-lattice chain in the $F$-vector space $\bar{V}^{i}$ defined by (40). Let $m_{i}$ denote the number of representations $\pi_{l}(1 \leq l \leq m)$ with endo-class $\theta_{i}$. Then $\overline{\mathcal{L}}^{i}$, considered as an $\mathfrak{o}_{F^{-}}$lattice chain, has period $e_{i}:=e\left(\overline{\mathcal{L}}^{i}\right)=e\left(E_{i} \mid F\right) m_{i}$, and $\overline{\mathcal{L}}_{\text {max }}^{i}$ has period $e\left(\overline{\mathcal{L}}_{\text {max }}^{i}\right)=m_{i}$.

Then let $\Lambda^{i}$ (resp. $\Lambda_{\max }^{i}$ ) denote the (strict) lattice sequence defined by the lattice chain $\overline{\mathcal{L}}^{i}$ (resp. $\overline{\mathcal{L}}_{\text {max }}^{i}$ ), considered as $\mathfrak{o}_{F}$-lattice chains. Then, using the addition of lattice sequences recalled in (15), we define

$$
\Lambda:=\Lambda^{1} \oplus \Lambda^{2} \oplus \cdots \oplus \Lambda^{q}
$$

and

$$
\Lambda_{\max }:=e\left(E_{1} \mid F\right) \Lambda_{\max }^{1} \oplus e\left(E_{2} \mid F\right) \Lambda_{\max }^{2} \oplus \cdots \oplus e\left(E_{q} \mid F\right) \Lambda_{\max }^{q}
$$


Let $\mathcal{L}_{\Lambda}, \mathcal{L}_{\Lambda_{\max }}$ denote the $\mathfrak{o}_{F}$-lattice chains attached to the lattice sequences $\Lambda, \Lambda_{\max }$, respectively, as in (11). Let $\mathfrak{A}_{\Lambda}, \mathfrak{A}_{\Lambda_{\max }}$ denote the hereditary $\mathfrak{o}_{F}$-orders in $A$ defined by the lattice chain $\mathcal{L}_{\Lambda}, \mathcal{L}_{\Lambda_{\max }}$, respectively. We have (see [10, Proposition 2.3. (i)]):

$$
\mathfrak{A}_{\Lambda}=\mathfrak{A}\left(\mathcal{L}_{\Lambda}\right)=\mathfrak{a}_{0}(\Lambda) \text { and } \quad \mathfrak{A}_{\Lambda_{\max }}=\mathfrak{A}\left(\mathcal{L}_{\Lambda_{\max }}\right)=\mathfrak{a}_{0}\left(\Lambda_{\max }\right)
$$

where $\mathfrak{a}_{0}(\Lambda), \mathfrak{a}_{0}\left(\Lambda_{\max }\right)$ are defined as in (8)).

Lemma 8. The $\mathfrak{o}_{F}$-order $\mathfrak{A}_{\Lambda}$ is contained in the $\mathfrak{o}_{F}$-order $\mathfrak{A}_{\Lambda_{\max }}$.

Proof. Let $e:=\operatorname{lcm}\left\{e_{1}, \ldots, e_{q}\right\}$. Both $\Lambda$ and $\Lambda_{\max }$ have period $e$. ¿From (15), we have

$$
\Lambda(e x)=\Lambda^{1}\left(e_{1} x\right) \oplus \cdots \oplus \Lambda^{q}\left(e_{q} x\right), \quad \Lambda_{\max }(e x)=\Lambda_{\max }^{1}\left(e_{1} x\right) \oplus \cdots \oplus \Lambda_{\max }^{q}\left(e_{q} x\right),
$$

for each $x \in \mathbb{R}$. On the other side, (12) gives

$$
\Lambda^{i}\left(\frac{e_{i}}{e} j\right)=\Lambda\left(l_{i}(j)\right) \quad \text { and } \quad \Lambda_{\max }^{i}\left(\frac{e_{i}}{e} j\right)=\Lambda_{\max }\left(l_{i}(j)\right)
$$

for each $j \in \mathbb{Z}$, where $l_{i}(j)$ is the integer defined by the relation

$$
l_{i}(j)-1<\frac{e_{i}}{e} j \leq l_{i}(j) .
$$

Hence

$\Lambda(j)=\Lambda^{1}\left(l_{1}(j)\right) \oplus \cdots \oplus \Lambda^{q}\left(l_{q}(j)\right), \quad \Lambda_{\max }(j)=\Lambda_{\max }^{1}\left(l_{1}(j)\right) \oplus \cdots \oplus \Lambda_{\max }^{q}\left(l_{q}(j)\right)$.

Then the result is consequence of Lemma 6.

The following definition generalizes Definitions 4 and 5 .

Definition 6. We set

$$
\mathfrak{A}^{\mathfrak{s}}:=\mathfrak{A}_{\Lambda_{\max }}, \quad \text { and } \quad J^{\mathfrak{s}}:=U\left(\mathfrak{A}^{\mathfrak{s}}\right)
$$

Example 2. We assume here that $q=m$, that is, the representations $\pi_{l}$ have all distinct endo-classes. It implies that $\bar{M}=\widetilde{M}=M$. Then each lattice sequence $\Lambda_{\max }^{l}$ has period 1 , and so $\Lambda_{\max }$ has also period 1 . We get in this case $J^{\mathfrak{s}}=\operatorname{GL}\left(N, \mathfrak{o}_{F}\right)$.

Theorem 6. There exists a $G$-cover $(J, \tau)$ of $\left(J_{M}, \lambda_{M}\right)$ such that

- $J \subset J^{\mathfrak{s}}$, 
- $\alpha:=\operatorname{Ind}_{J}^{J^{\mathfrak{s}}}(\tau)$ is irreducible. Hence $\left(J^{\mathfrak{s}}, \alpha\right)$ is an $\mathfrak{s}$-type.

Proof. Let $(J, \tau)$ be the $G$-cover of $(\bar{K}, \bar{\tau})$ constructed in the similar way as in [10, $\S 8]$, in particular, we have

$$
J \subset U\left(\mathfrak{A}_{\Lambda}\right) .
$$

Then the first assertion follows from Lemma 8 .

On the other side the same proof as those of [10, §8.2, Main Theorem] gives the following formula for the intertwining:

$$
\mathcal{I}_{G}(\tau)=J \cdot \mathcal{I}_{\widetilde{M}}\left(\tau_{\widetilde{M}}\right) \cdot J .
$$

Since $J \subset J^{\mathfrak{s}}$, it implies:

$$
\mathcal{I}_{J^{\mathfrak{s}}}(\tau)=J \cdot \mathcal{I}_{\widetilde{M} \cap J^{\mathfrak{s}}}\left(\tau_{\widetilde{M}}\right) \cdot J=J \cdot \mathcal{I}_{\widetilde{J}^{\mathfrak{s}}}(\widetilde{\tau}) \cdot J .
$$

Now, by Lemma 5 , we have

$$
\mathcal{I}_{\widetilde{J^{\mathfrak{s}}}}\left(\tau_{\widetilde{M}}\right)=\widetilde{K}
$$

We get

$$
\mathcal{I}_{J^{s}}(\tau)=J,
$$

and the result follows from Proposition 2

\section{Supercuspidal Bernstein components}

Let $\mathfrak{s}=[G, \pi]_{G}$, where $G=\mathrm{GL}(N, F)$. Here $\pi$ is an irreducible supercuspidal representation of $G$.

Let $(J, \lambda)$ be a maximal simple type contained in $\pi$, as in [8. We have $e=1$ and hence $\mathfrak{A}_{\mathfrak{s}}=\mathrm{M}\left(N, \mathfrak{o}_{F}\right)$. It follows that $J^{\mathfrak{s}}=\operatorname{GL}\left(N, \mathfrak{o}_{F}\right)=L_{0}$. By Proposition 4 , the representation $\alpha=\operatorname{Ind}_{J}^{L_{0}}(\lambda)$ is irreducible. The pair $\left(L_{0}, \alpha\right)$ is an $\mathfrak{s}$-type. The restriction to $L_{0}$ of a smooth irreducible representation $\pi^{\prime}$ of $G$ contains $\alpha$ if and only if $\pi^{\prime}$ is isomorphic to $\pi \otimes \chi \circ$ det, where $\chi$ is an unramified quasicharacter of $F^{\times}$. Moreover, $\pi$ contains $\alpha$ with multiplicity 1 . In fact, the representation $\alpha$ is the unique smooth irreducible representation $\tau$ of $L_{0}$ such that $\left(L_{0}, \tau\right)$ is an $\mathfrak{s}$-type, see [16].

The little complex $C_{*}(\mathfrak{s})$ determined by $\alpha$ is

$$
0 \longleftarrow C_{0}(\mathfrak{s}) \longleftarrow 0
$$

where $C_{0}(\mathfrak{s})$ is the free abelian group on the invariant 0-cycle

$$
\left(\tau, \mathcal{R}(\tau), \mathcal{R}^{2}(\tau), \ldots, \mathcal{R}^{n-1}(\tau)\right)
$$


The total homology of the little complex is given by $h_{0}(\mathfrak{s})=\mathbb{Z}$. Therefore, by Lemma 1 we have

$$
H_{\text {ev }}(\mathfrak{s})=\mathbb{Z}=H_{\text {odd }}(\mathfrak{s}) .
$$

Theorem 7. Let $\pi$ be an irreducible unitary supercuspidal representation of $\operatorname{GL}(N)$. Let $\mathfrak{s}=[G, \pi]_{G}$. Then we have

$$
H_{\text {ev }}(\mathfrak{s}) \cong K_{0}\left(\mathcal{A}^{\mathfrak{s}}\right), \quad H_{\text {odd }}(\mathfrak{s}) \cong K_{1}\left(\mathcal{A}^{\mathfrak{s}}\right) .
$$

Proof. The $C^{*}$-ideal $\mathcal{A}^{\mathfrak{s}}$ is given by

$$
\mathcal{A}^{\mathfrak{s}}=C\left(S^{1}, \mathfrak{K}\right)
$$

where $\mathfrak{K}$ is the $C^{*}$-algebra of compact operators and

$$
S^{1}=\left\{\pi \otimes \chi \circ \operatorname{det}: \chi \in\left(F^{\times}\right)^{\wedge}\right\} .
$$

The noncommutative $C^{*}$-algebra $\mathcal{A}^{\mathfrak{s}}$ is strongly Morita equivalent to the commutative $C^{*}$-algebra $C\left(S^{1}\right)$. For this $C^{*}$-algebra we have

$$
K_{j}\left(C\left(S^{1}\right)\right) \cong K^{j}\left(S^{1}\right)=\mathbb{Z}
$$

where $j=0,1$.

\section{Generic Bernstein components attached to a maximal Levi subgroup}

We assume in this section that $\mathfrak{s}=[M, \sigma]_{G}$ with $M \cong \mathrm{GL}\left(N_{1}\right) \times \mathrm{GL}\left(N_{2}\right)$ a 2 blocks Levi subgroup of $G$ such that $W_{\mathfrak{t}}=\{1\}$. Note that the last conditions is always satisfied if $N_{1} \neq N_{2}$.

Let $\left(J_{M}, \lambda_{M}\right)$ be an $\mathfrak{t}$-type and let $(J, \tau)$ be the $G$-cover of $\left(J_{M}, \tau_{M}\right)$ considered in Theorem [6. We have shown there that $\alpha:=\operatorname{Ind}_{J}^{J^{5}}(\tau)$ is irreducible. It then follows from Propositions 2 and 5 that $\beta=\operatorname{Ind}_{J}^{L_{0}}(\tau)$ is irreducible.

Let $C_{0}(\tau), C_{1}(\tau)$ denote respectively the free abelian group on one generator $\left(\beta, \mathcal{R}(\beta), \ldots, \mathcal{R}^{N-1}(\beta)\right.$, and on $\left(\alpha, \mathcal{R}(\alpha), \ldots, \mathcal{R}^{N-1} \alpha\right)$. The little complex is

$$
0 \longleftarrow C_{0}(\mathfrak{s}) \stackrel{\partial}{\longleftarrow} C_{1}(\mathfrak{s}) \longleftarrow 0
$$

The map $\partial$ is 0 by vertex compatibility of $\left(\alpha, \mathcal{R}(\alpha), \ldots, \mathcal{R}^{N-1}(\alpha)\right)$. Then $h_{0}(\mathfrak{s})=\mathbb{Z}, h_{1}(\mathfrak{s})=\mathbb{Z}$ and so $H_{\mathrm{ev}}(\mathfrak{s})=\mathbb{Z}^{2}=H_{\text {odd }}(\mathfrak{s})$.

The subset of the tempered dual of $\mathrm{GL}(N)$ which contains the $\mathfrak{s}$-type $(J, \tau)$ has the structure of a compact 2-torus. But $K^{0}\left(\mathbb{T}^{2}\right)=\mathbb{Z}^{2}=K^{1}\left(\mathbb{T}^{2}\right)$ as required. 
Theorem 8. The $\mathfrak{s}$-type $(J, \tau)$ generates a little complex $C(\mathfrak{s})$. For this complex we have

$$
H_{\mathrm{ev}}(\mathfrak{s}) \cong K_{0}\left(\mathcal{A}^{\mathfrak{s}}\right)=\mathbb{Z}^{2}, \quad H_{\text {odd }}(\mathfrak{s}) \cong K_{1}\left(\mathcal{A}^{\mathfrak{s}}\right)=\mathbb{Z}^{2}
$$

Note that the above Theorem applies to the intermediate principal series of GL(3). In the next section, we will consider the principal series of GL(3).

\section{Principal series in $\mathrm{GL}(3)$}

Here $s_{0}, s_{1}, s_{2}$ are the standard involutions

$$
s_{1}=\left(\begin{array}{ccc}
0 & 1 & 0 \\
1 & 0 & 0 \\
0 & 0 & 1
\end{array}\right) \quad s_{2}=\left(\begin{array}{ccc}
1 & 0 & 0 \\
0 & 0 & 1 \\
0 & 1 & 0
\end{array}\right) \quad s_{0}=\left(\begin{array}{ccc}
0 & 0 & \varpi^{-1} \\
0 & 1 & 0 \\
\varpi & 0 & 0
\end{array}\right)
$$

where

$$
\Pi=\Pi_{3}=\left(\begin{array}{ccc}
0 & 1 & 0 \\
0 & 0 & 1 \\
\varpi & 0 & 0
\end{array}\right) .
$$

Note that $\operatorname{val}(\operatorname{det}(\Pi))=1$. Restricted to the affine line $\mathbb{R}$ in the enlarged building $\beta^{1} \mathrm{GL}(3)=\beta \mathrm{SL}(3) \times \mathbb{R}$, $\Pi$ sends $t$ to $t+1$. We also have $\Pi^{3}=\varpi 1 \epsilon$ GL(3).

We have the double coset identities

$$
\begin{gathered}
0 \leq k \leq 2 \Longrightarrow I \backslash J_{k} / I=\left\{1, s_{k}\right\} \\
r, s, t \text { distinct } \Longrightarrow J_{r} \backslash L_{s} / J_{r}=\left\{1, s_{t}\right\} .
\end{gathered}
$$

Let $\mathfrak{s}=[T, \sigma]_{G}$, where $T$ is the diagonal split torus in GL(3):

$$
T=\left(\begin{array}{ccc}
F^{\times} & 0 & 0 \\
0 & F^{\times} & 0 \\
0 & 0 & F^{\times}
\end{array}\right)
$$

and $\sigma$ is an irreducible smooth character of $T$.

\subsection{Construction of an $\mathfrak{s - t y p e , ~ f o l l o w i n g ~ R o c h e ~}$}

For $u \in F$, we set

$$
x_{1,2}(u)=\left(\begin{array}{ccc}
1 & u & 0 \\
0 & 1 & 0 \\
0 & 0 & 1
\end{array}\right), \quad x_{1,3}(u)=\left(\begin{array}{ccc}
1 & 0 & u \\
0 & 1 & 0 \\
0 & 0 & 1
\end{array}\right), \quad x_{2,3}(u)=\left(\begin{array}{ccc}
1 & 0 & 0 \\
0 & 1 & u \\
0 & 0 & 1
\end{array}\right) \text {, }
$$




$$
x_{2,1}(u)=\left(\begin{array}{ccc}
1 & 0 & 0 \\
u & 1 & 0 \\
0 & 0 & 1
\end{array}\right), \quad x_{3,1}(u)=\left(\begin{array}{ccc}
1 & 0 & 0 \\
0 & 1 & 0 \\
u & 0 & 1
\end{array}\right), \quad x_{3,2}(u)=\left(\begin{array}{lll}
1 & 0 & 0 \\
0 & 1 & 0 \\
0 & u & 1
\end{array}\right)
$$

and, for any $k \in \mathbb{Z}$,

$$
U_{i, j, k}=x_{i, j}\left(\mathfrak{p}_{F}^{k}\right)
$$

Let $\Phi=\left\{\alpha_{i, j}: 1 \leq i, j \leq 2\right\}$ be the set of roots of $G$ with respect to $T$. For each root $\alpha_{i, j}$, let $\alpha_{i, j}^{\vee}$ denotes the corresponding coroot. We have

$$
\begin{array}{rlrl}
\alpha_{1,2}^{\vee}(t) & =\left(\begin{array}{ccc}
t^{-1} & 0 & 0 \\
0 & t & 0 \\
0 & 0 & 1
\end{array}\right), & \alpha_{2,1}^{\vee}(t)=\left(\begin{array}{ccc}
t & 0 & 0 \\
0 & t^{-1} & 0 \\
0 & 0 & 1
\end{array}\right), \\
\alpha_{1,3}^{\vee}(t)=\left(\begin{array}{ccc}
t^{-1} & 0 & 0 \\
0 & 1 & 0 \\
0 & 0 & t
\end{array}\right), & \alpha_{3,1}^{\vee}(t)=\left(\begin{array}{ccc}
t & 0 & 0 \\
0 & 1 & 0 \\
0 & 0 & t^{-1}
\end{array}\right), \\
\alpha_{2,3}^{\vee}(t)=\left(\begin{array}{ccc}
1 & 0 & 0 \\
0 & t^{-1} & 0 \\
0 & 0 & t
\end{array}\right), & \alpha_{3,2}^{\vee}(t)=\left(\begin{array}{ccc}
1 & 0 & 0 \\
0 & t & 0 \\
0 & 0 & t^{-1}
\end{array}\right) .
\end{array}
$$

Define $\sigma: T \rightarrow \mathbb{T}$ by

$$
\sigma\left(\begin{array}{lll}
a & 0 & 0 \\
0 & b & 0 \\
0 & 0 & c
\end{array}\right)=\sigma_{1}(a) \sigma_{2}(b) \sigma_{3}(c)
$$

where $\sigma_{i}: F^{\times} \rightarrow \mathbb{T}$ is a character of $F^{\times}$, for $i=1,2,3$.

Hence $\sigma \circ \alpha_{i, j}^{\vee}: \mathfrak{o}_{F}^{\times} \rightarrow \mathbb{T}$ is the smooth character of $\mathfrak{o}_{F}^{\times}$defined by

$$
\sigma \circ \alpha_{i, j}^{\vee}(t)=\sigma_{j}(t) \sigma_{i}\left(t^{-1}\right)=\left(\sigma_{j} \sigma_{i}^{-1}\right)(t)
$$

Now if $\chi: \mathfrak{o}_{F}^{\times} \rightarrow \mathbb{T}$ is a smooth character, let $c(\chi)$ be the conductor of $\chi$ : the least integer $n \geq 1$ such that $1+\mathfrak{p}_{F}^{n} \subset \operatorname{ker}(\chi)$. We will write $c_{i, j}$ for $c\left(\sigma \circ \alpha_{i, j}^{\vee}\right)$. We get

$$
c_{i, j}=c\left(\sigma_{j} / \sigma_{i}\right)=c_{j, i} .
$$

We can define a function $f=f_{\sigma}: \Phi \rightarrow \mathbb{Z}$ (here $\Phi$ is the set of roots) as follows:

$$
f_{\sigma}\left(\alpha_{i, j}\right)= \begin{cases}{\left[c_{i, j} / 2\right]} & \text { if } \alpha_{i, j} \in \Phi^{+} \\ {\left[\left(c_{i, j}+1\right) / 2\right]} & \text { if } \alpha_{i, j} \in \Phi^{-}\end{cases}
$$

Here $[x]$ denotes the largest integer $\leq x$. 
Let

$$
U_{\sigma}=\left\langle U_{i, j, f\left(\alpha_{i, j}\right)}: \alpha_{i, j} \in \Phi\right\rangle,
$$

and

$$
J=\left\langle{ }^{\circ} T, U_{\sigma}\right\rangle={ }^{\circ} T U_{\sigma}=U_{\sigma}{ }^{\circ} T
$$

where ${ }^{\circ} T$ is the compact part of $T$,

$$
{ }^{\circ} T=\left(\begin{array}{ccc}
\mathfrak{o}_{F}^{\times} & 0 & 0 \\
0 & \mathfrak{o}_{F}^{\times} & 0 \\
0 & 0 & \mathfrak{o}_{F}^{\times}
\end{array}\right) .
$$

It follows that

$$
J=\left(\begin{array}{ccc}
\mathfrak{o}_{F}^{\times} & \mathfrak{p}_{F}^{\left[c_{1,2} / 2\right]} & \mathfrak{p}_{F}^{\left[c_{1,3} / 2\right]} \\
\mathfrak{p}_{F}^{\left[\left(c_{1,2}+1\right) / 2\right]} & \mathfrak{o}_{F}^{\times} & \mathfrak{p}_{F}^{\left[c_{2,3} / 2\right]} \\
\mathfrak{p}_{F}^{\left[\left(c_{1,3}+1\right) / 2\right]} & \mathfrak{p}_{F}^{\left[\left(c_{2,3}+1\right) / 2\right]} & \mathfrak{o}_{F}^{\times}
\end{array}\right)
$$

The group $J$ will give the open compact group we are looking for.

Next, we need to figure out what is the correct character of $J$. In order to do that, we set

$$
T_{\sigma}=\prod_{\alpha_{i, j} \in \Phi} \alpha_{i, j}^{\vee}\left(1+\mathfrak{p}_{F}^{f\left(\alpha_{i, j}\right)+f\left(-\alpha_{i, j}\right)}\right) \subset{ }^{\circ} T .
$$

Setting

$$
U_{\sigma}^{+}=U_{\sigma} \cap\left(\begin{array}{ccc}
1 & F & F \\
0 & 1 & F \\
0 & 0 & 1
\end{array}\right) \quad \text { and } \quad U_{\sigma}^{-}=U_{\sigma} \cap\left(\begin{array}{ccc}
1 & 0 & 0 \\
F & 1 & 0 \\
F & F & 1
\end{array}\right),
$$

we obtain

$$
U_{\sigma}=U_{\sigma}^{-} \cdot T_{\sigma} \cdot U_{\sigma}^{+} \quad \text { and } \quad J=U_{\sigma}^{-} \cdot{ }^{\circ} T \cdot U_{\sigma}^{+} .
$$

It follows that

$$
J / U_{\sigma} \cong{ }^{\circ} T / T_{\sigma} .
$$

By construction, $T_{\sigma} \subset \operatorname{ker}\left(\sigma_{\left.\right|^{\circ} T}\right)$. Hence $\sigma_{{ }^{\circ} T}$ defines a character of ${ }^{\circ} T / T_{\sigma}$, and so can be lifted to a character $\tau$ of $J$. Then $(J, \tau)$ an $\mathfrak{s}$-type by [18, Theorem 7.7].

\subsection{Intertwining}

We first recall that the following results ([18, Theorem 4.15])

$$
I_{G}(\tau)=J \widetilde{W}(\sigma) J,
$$


where

$$
\widetilde{W}(\sigma)=\left\{v \in \widetilde{W}:{ }^{v} \sigma=\sigma\right\} .
$$

More generally, it follows by the same proof as those of [18, Theorem 4.15], using [1, Prop. 9.3] instead of [18, Prop. 4.11], that, for each $w \in W$,

$$
I_{G}\left(\tau,{ }^{\tau}\right)=J \widetilde{W}\left(\sigma,{ }^{w} \sigma\right)^{w} J
$$

where

$$
\widetilde{W}\left(\sigma,{ }^{w} \sigma\right)=\left\{v \in \widetilde{W}:{ }^{v} \sigma={ }^{w} \sigma\right\}
$$

Let

$$
\Phi(\sigma)=\left\{\alpha_{i, j} \in \Phi:\left.\left(\sigma_{i}\right)\right|_{F} ^{\times}=\left(\sigma_{j}\right) \mid \mathfrak{o}_{F}^{\times}\right\} \subset \Phi .
$$

The group $W_{0}(\sigma)$ is equal to the group $W_{\mathfrak{s}_{T}}$, where $\mathfrak{s}_{T}=[T, \lambda]_{T}$. We observe that

$$
I_{L_{0}}(\tau)=J W_{0}(\sigma) J
$$

9.2.1 The case $\Phi(\sigma)=\Phi$. Let $\mathfrak{s}=[T, \sigma]_{G}$, where $\sigma=\psi \circ$ det with $\psi$ a smooth character of $F^{\times}$. In this case $c_{i, j}=1$ for any $i, j$. It follows that $J=I$.

The pair $(I, \tau)$ is an $\mathfrak{s}$-type. We will construct cycles from this type. It follows from (57) that, as $\mathbb{C}$-algebras,

$$
\operatorname{End}_{L_{0}}\left(\operatorname{Ind}_{I}^{L_{0}} \tau\right) \cong \mathcal{H}\left(\mathrm{GL}\left(3, k_{F}\right) / / B\right) .
$$

We also have, as $\mathbb{C}$-algebras,

$$
\mathcal{H}\left(\mathrm{GL}\left(3, k_{F}\right) / / B\right) \cong \mathbb{C}\left[W_{0}\right] \cong \mathbb{C} \oplus \mathbb{C} \oplus M_{2}(\mathbb{C})
$$

so that

$$
\operatorname{Ind}_{I}^{L_{0}} \tau=\lambda_{L_{0}} \oplus \mu_{L_{0}} \oplus \nu_{L_{0}} \oplus \nu_{L_{0}}
$$

where $\lambda_{L_{0}}, \mu_{L_{0}}, \nu_{L_{0}}$ are distinct.

We also have

$$
\sigma \mid J_{0} \hookrightarrow \operatorname{Ind}_{I}^{J_{0}} \tau
$$

by Frobenius reciprocity. The triple $\left(\sigma \mid J_{0}, \mathcal{R}\left(\sigma \mid J_{0}\right), \mathcal{R}^{2}\left(\sigma \mid J_{0}\right)\right)$ is an invariant 1-cycle, and is not the boundary of $1_{I}$.

We now form the little complex:

- $C_{0}(\mathfrak{s})$ is the free abelian group on the three invariant 0-cycles

$$
\begin{aligned}
\lambda_{L} & :=\left(\lambda_{L_{0}}, \mathcal{R}\left(\lambda_{L_{0}}\right), \mathcal{R}^{2}\left(\lambda_{L_{0}}\right)\right) \\
\mu_{L} & :=\left(\mu_{L_{0}}, \mathcal{R}\left(\mu_{L_{0}}\right), \mathcal{R}^{2}\left(\mu_{L_{0}}\right)\right) \\
\nu_{L} & :=\left(\nu_{L_{0}}, \mathcal{R}\left(\nu_{L_{0}}\right), \mathcal{R}^{2}\left(\nu_{L_{0}}\right)\right)
\end{aligned}
$$


- $C_{1}(\mathfrak{s})$ is the free abelian group on the invariant 1-cycle

$$
\lambda_{J}:=\left(\sigma \mid J_{0}, \mathcal{R}\left(\sigma \mid J_{0}\right), \mathcal{R}^{2}\left(\sigma \mid J_{0}\right)\right)
$$

In the little complex

$$
0 \longleftarrow C_{0}(\mathfrak{s}) \stackrel{0}{\longleftarrow} C_{1}(\mathfrak{s}) \longleftarrow 0
$$

we have

$$
h_{0}(\mathfrak{s})=\mathbb{Z}^{3}, h_{1}(\mathfrak{s})=\mathbb{Z} .
$$

The total homology of the little complex is $\mathbb{Z}^{4}$. As generating cycles we may take

$$
\lambda_{L}, \mu_{L}, \nu_{L}, \lambda_{J}
$$

and so, by Lemma 1, the even (resp. odd) chamber homology groups are

$$
H_{\text {ev }}(\mathfrak{s})=\mathbb{Z}^{4}, \quad H_{\text {odd }}(\mathfrak{s})=\mathbb{Z}^{4} .
$$

Each irreducible representation $\rho$ of a compact open subgroup $J$ creates an idempotent in $\mathcal{A}$ as follows. Let $d$ denote the dimension of $\rho$, let $\chi$ denote the character of $\rho$. Form the function $d \cdot \chi: J \longrightarrow \mathbb{C}$ and extend by 0 to $G$. This function on $G$ is a non-zero idempotent in $\mathcal{A}$, with the convolution product. We will denote this idempotent by $e(\rho)$ :

$$
e(\rho) * e(\rho)=e(\rho) .
$$

The inclusion

$$
H_{\mathrm{ev}}(\mathfrak{s}) \hookrightarrow K_{0}(\mathcal{A})
$$

is given explicitly as follows:

$$
\lambda_{L} \mapsto e\left(\lambda_{L_{1}}\right), \mu_{L} \mapsto e\left(\mu_{L_{1}}\right), \nu_{L} \mapsto e\left(\nu_{L_{1}}\right), \lambda_{J} \mapsto e\left(\lambda_{J_{1}}\right) .
$$

It follows from [17] that the $C^{*}$-ideal $\mathcal{A}^{\mathfrak{s}}$ is given as follows:

$$
\mathcal{A}^{\mathfrak{s}} \cong C\left(\mathrm{Sym}^{3} \mathbb{T}, \mathfrak{K}\right) \oplus C\left(\mathbb{T}^{2}, \mathfrak{K}\right) \oplus C(\mathbb{T}, \mathfrak{K}) .
$$

The symmetric cube $\operatorname{Sym}^{3} \mathbb{T}$ is homotopy equivalent to $\mathbb{T}$ via the product map

$$
\operatorname{Sym}^{3} \mathbb{T} \sim \mathbb{T}, \quad\left(z_{1}, z_{2}, z_{3}\right) \mapsto z_{1} z_{2} z_{3} .
$$

Hence $K_{0}\left(\mathcal{A}^{\mathfrak{s}}\right)=\mathbb{Z}^{4}=K_{1}\left(\mathcal{A}^{\mathfrak{s}}\right)$ as required.

Note that

- $\mathrm{Sym}^{3} \mathbb{T}$ is in the minimal unitary principal series of $\mathrm{GL}(3)$ 
- $\mathbb{T}^{2}$ is in the intermediate unitary principal series of $\mathrm{GL}(3)$

- $\mathbb{T}$ is in the discrete series of GL(3); if $\tau=1$ then $\mathbb{T}$ comprises the unramified unitary twists of the Steinberg representation of GL(3)

These are precisely the tempered representations of GL(3) which contain the type $(I, \tau)$.

Theorem 9. Let $\mathfrak{s}=[T, \sigma]_{G}$ where $\sigma=\psi \circ \operatorname{det}$ and $\psi$ is a smooth (unitary) character of $F^{\times}$. Then we have

$$
H_{\mathrm{ev}}(\mathfrak{s}) \cong K_{0}\left(\mathcal{A}^{\mathfrak{s}}\right)=\mathbb{Z}^{4}, \quad H_{\text {odd }}(\mathfrak{s}) \cong K_{1}\left(\mathcal{A}^{\mathfrak{s}}\right)=\mathbb{Z}^{4} .
$$

9.2.2 The case $\emptyset \neq \Phi(\sigma) \neq \Phi$

Assume that $\left(\sigma_{1}\right)_{\mid \mathfrak{o}_{F}^{\times}}=\left(\sigma_{2}\right)_{\mid \mathfrak{o}_{F}^{\times}} \neq\left(\sigma_{3}\right)_{\mid \mathfrak{o}_{F}^{\times}}$. We have

$$
J=\left(\begin{array}{ccc}
\mathfrak{o}_{F}^{\times} & \mathfrak{o}_{F} & \mathfrak{p}_{F}^{[\ell / 2]} \\
\mathfrak{p}_{F} & \mathfrak{o}_{F}^{\times} & \mathfrak{p}_{F}^{\ell / 2]} \\
\mathfrak{p}_{F}^{[(\ell+1) / 2]} & \mathfrak{p}_{F}^{[(\ell+1) / 2]} & \mathfrak{o}_{F}^{\times}
\end{array}\right),
$$

where $\ell=c_{1,3}=c_{2,3}$, and

$$
\tau\left(\begin{array}{lll}
a & * & * \\
* & b & * \\
* & * & c
\end{array}\right)=\sigma_{1}(a) \sigma_{1}(b) \sigma_{3}(c) .
$$

It is clear that $s_{1} \in I_{L_{0}}(\tau)$. The Weyl group $W_{\mathfrak{s}_{T}}=\mathbb{Z} / 2 \mathbb{Z}$ and so we have $I_{L_{o}}(\tau)=J \cup J s_{1} J$. The complete list is as follows:

$$
\begin{gathered}
I_{I}(\tau)=J \\
I_{J_{1}}(\tau)=J<s_{1}>J, \quad I_{J_{2}}(\tau)=J, \quad I_{J_{0}}(\tau)=J \\
I_{L_{1}}(\tau)=J<s^{\prime}>J, \quad I_{L_{2}}(\tau)=J<s_{1}>J, \quad I_{L_{0}}(\tau)=J<s_{1}>J
\end{gathered}
$$

where

$$
s^{\prime}=\left(\begin{array}{lll}
0 & \varpi^{-1} & 0 \\
\varpi & 0 & 0 \\
0 & 0 & 1
\end{array}\right)
$$

Lemma 9. Let $\tau_{1}=\operatorname{Ind}_{J}^{I}(\tau)$. Then $\tau_{1}$ is irreducible.

Proof. This follows from proposition 21 since $I_{I}(\tau)=J$. It follows that $\left(I, \tau_{1}\right)$ is an $\mathfrak{s}$-type. 
Lemma 10. We have

$$
\operatorname{Ind}_{I}^{J_{1}} \tau_{1}=\xi_{1} \oplus \eta_{1}, \quad \operatorname{Ind}_{I}^{L_{0}} \tau_{1}=\gamma_{0} \oplus \delta_{0}
$$

Proof. We have $I_{J_{1}}(\tau)=J \cup s_{1} J$. Hence

$$
\operatorname{End}_{J_{1}}\left(\operatorname{Ind}_{I}^{J_{1}} \tau_{1}\right)=\mathcal{I}_{1}(\tau) \oplus \mathcal{I}_{s_{1}}(\tau)=\mathbb{C} \oplus \mathbb{C}
$$

This implies that $\operatorname{Ind}_{I}^{J_{1}} \tau_{1}$ has two distinct irreducible constituents $\xi_{1}, \eta_{1}$. Now, we replace $J_{1}$ by $L_{0}$, and infer that $\operatorname{Ind}_{I}^{L_{0}} \tau_{1}$ has two distinct irreducible constituents $\gamma_{0}, \delta_{0}$.

It follows that

$$
\begin{gathered}
\operatorname{Ind}_{I}^{J_{2}} \mathcal{R}\left(\tau_{1}\right)=\mathcal{R}\left(\xi_{1}\right) \oplus \mathcal{R}\left(\eta_{1}\right), \\
\operatorname{Ind}_{I}^{J_{0}} \mathcal{R}^{2}\left(\tau_{1}\right)=\mathcal{R}^{2}\left(\xi_{1}\right) \oplus \mathcal{R}^{2}\left(\eta_{1}\right) .
\end{gathered}
$$

This creates two invariant 1-chains

$$
\xi:=\left(\xi_{1}, \mathcal{R}\left(\xi_{1}\right), \mathcal{R}^{2}\left(\xi_{1}\right)\right), \eta:=\left(\eta_{1}, \mathcal{R}\left(\eta_{1}\right), \mathcal{R}^{2}\left(\eta_{1}\right)\right) .
$$

It follows from (5) that

$$
\begin{gathered}
\operatorname{Ind}_{I}^{L_{0}} \tau_{1} \cong \operatorname{Ind}_{I}^{L_{0}} \mathcal{R}\left(\tau_{1}\right) \\
\zeta_{1}:=\operatorname{Ind}_{I}^{J_{1}} \mathcal{R}\left(\tau_{1}\right) \cong \operatorname{Ind}_{I}^{J_{1}} \mathcal{R}^{2}\left(\tau_{1}\right)
\end{gathered}
$$

By (17) we have

$$
0=\left\langle\operatorname{Ind}_{J}^{J_{1}} \tau, \operatorname{Ind}_{J}^{J_{1}} \mathcal{R}(\tau)\right\rangle
$$

Let $C_{0}(\mathfrak{s})$ be the free abelian group generated by the two invariant 0-cycles

$$
\left(\gamma_{0}, \mathcal{R}\left(\gamma_{0}\right), \mathcal{R}^{2}\left(\gamma_{0}\right)\right),\left(\delta_{0}, \mathcal{R}\left(\delta_{0}\right), \mathcal{R}^{2}\left(\delta_{0}\right)\right)
$$

Let $C_{1}(\mathfrak{s})$ be the free abelian group generated by the two invariant 1-cycles $\xi$ and $\zeta$.

The little complex is then

$$
0 \longleftarrow C_{0}(\mathfrak{s}) \stackrel{0}{\longleftarrow} C_{1}(\mathfrak{s}) \longleftarrow 0
$$

We have $h_{0}(\mathfrak{s})=\mathbb{Z}^{2}, h_{1}(\mathfrak{s})=\mathbb{Z}^{2}$ and the total homology is $\mathbb{Z}^{4}$ and so $H_{\text {ev }}(\mathfrak{s})=\mathbb{Z}^{4}=H_{\text {odd }}(\mathfrak{s})$.

The definition of $\zeta:=\left(\zeta_{0}, \mathcal{R}\left(\zeta_{0}\right), \mathcal{R}^{2}\left(\zeta_{0}\right)\right)$ shows that

$$
\partial\left(\tau_{1}+\mathcal{R}\left(\tau_{1}\right)+\mathcal{R}^{2}\left(\tau_{1}\right)\right)=\xi+\eta+2 \zeta
$$


so that $\eta$ and $-(\xi+2 \zeta)$ are homologous. Therefore the invariant 1-cycle $\eta$ does not contribute a new homology class in $H_{1}\left(G ; \beta^{1} G\right)$.

The $C^{*}$-ideal $\mathcal{A}^{\mathfrak{s}}$ is as follows:

$$
C\left(\mathbb{T}^{2}, \mathfrak{K}\right) \oplus C\left(\mathrm{Sym}^{2} \mathbb{T} \times \mathbb{T}, \mathfrak{K}\right) .
$$

To identify these ideals, we proceed as follows. First, let $\Psi\left(F^{\times}\right)$denote the group of unramified unitary characters of $F^{\times}$. The first summand is determined by the compact orbit

$$
\mathcal{O}\left(\operatorname{St}\left(\sigma_{1}, 2\right) \otimes \sigma_{3}\right)=\left\{\chi_{1} \operatorname{St}\left(\sigma_{1}, 2\right) \otimes \chi_{2} \sigma_{3}: \chi_{j} \in \Psi\left(F^{\times}\right)\right\}
$$

where $\operatorname{St}\left(\sigma_{1}, 2\right)$ is a generalized Steinberg representation; the second is determined by the compact orbit

$$
\mathcal{O}\left(\sigma_{1} \otimes \sigma_{1} \otimes \sigma_{3}\right)=\left\{\chi_{1} \sigma_{1} \otimes \chi_{2} \sigma_{1} \otimes \chi_{3} \sigma_{3}: \chi_{j} \in \Psi\left(F^{\times}\right)\right\} .
$$

The compact space $\operatorname{Sym}^{2} \mathbb{T} \times \mathbb{T}$ is homotopy equivalent to the 2-torus $\mathbb{T}^{2}$.

The space $\operatorname{Sym}^{2} \mathbb{T} \times \mathbb{T}$ is in the minimal unitary principal series of GL(3) and the space $\mathbb{T}^{2}$ is in the intermediate unitary principal series of GL(3). The union of these two compact spaces is precisely the set of tempered representations of $\mathrm{GL}(3)$ which contain the $\mathfrak{s}$-type $(J, \tau)$.

The $K$-groups are now immediate:

$$
K_{j}\left(\mathcal{A}^{\mathfrak{s}}\right)=\mathbb{Z}^{4}
$$

with $j=0,1$.

Theorem 10. Let $\mathfrak{s}=[T, \sigma]_{G}$. We have

$$
H_{\mathrm{ev}}(\mathfrak{s}) \cong K_{0}\left(\mathcal{A}^{\mathfrak{s}}\right)=\mathbb{Z}^{4}, \quad H_{\text {odd }}(\mathfrak{s}) \cong K_{1}\left(\mathcal{A}^{\mathfrak{s}}\right)=\mathbb{Z}^{4}
$$

9.2.3 The case $\Phi(\sigma)=\emptyset$. The generic torus. The Bernstein component is $\left[T, \sigma_{1} \otimes \sigma_{2} \otimes \sigma_{3}\right]$. The Weyl group $W(T)=W_{0}=S_{3}$, and the associated parahoric subgroup is the Iwahori subgroup $I$.

The restrictions of $\sigma_{1}, \sigma_{2}$ and $\sigma_{3}$ to $\mathfrak{o}_{F}^{\times}$are all distinct. We have $\Phi(\sigma)=\emptyset$. We have $\widetilde{W}(\sigma)=D$, where $D$ is the subgroup of $T$ whose eigenvalues are powers of $\varpi$. The subgroup $D$ is free abelian of rank 3 . The only compact element in $D$ is $1_{G}$. The only double- $J$-coset representative in $L_{0}$ which $G$-intertwines $\tau$ is $1_{G}$. This proves the following:

Lemma 11. If $r=0,1,2$ then $\operatorname{Ind}_{J}^{J_{r}}(\tau)$ is irreducible, $\operatorname{Ind}_{J}^{L_{r}}(\tau)$ is irreducible.

Let $\alpha=\operatorname{Ind}_{J}^{I}(\tau)$. Then $\alpha$ is irreducible. Therefore $(I, \alpha)$ is an $\mathfrak{s}$-type. 
Lemma 12. If $w \in W_{0}$ then $\operatorname{Ind}_{I}^{L_{0}} \alpha=\operatorname{Ind}_{I}^{L_{0}}\left({ }^{w} \alpha\right)$.

Proof. We have $\operatorname{Ind}_{I}^{L_{0}}(\alpha)=\operatorname{Ind}_{J}^{L_{0}}(\tau)$ and $\operatorname{Ind}_{I}^{L_{0}}\left({ }^{w} \alpha\right)=\operatorname{Ind}_{J}^{L_{0}}\left({ }^{w} \tau\right)$. By Proposition 3, it is sufficient to prove that $I_{G}\left(\tau,{ }^{w} \tau\right) \neq\{0\}$. But $I_{G}\left(\tau,{ }^{w} \tau\right)=$ $J \widetilde{W}\left(\sigma,{ }^{w} \sigma\right) J$.

Lemma 13. If $w \in W_{0}$ then

$$
\operatorname{Ind}_{I}^{J_{r}}(\alpha) \cong \operatorname{Ind}_{I}^{J_{r}}\left({ }^{w} \alpha\right) \Longleftrightarrow w \in<s_{r}>
$$

with $0 \leq r \leq 2$.

Proof. By Proposition 3 ,

$$
\operatorname{Ind}_{J}^{J_{r}}(\tau) \cong \operatorname{Ind}_{J}^{J_{r}}\left({ }^{w} \tau\right) \Longleftrightarrow I_{J_{r}}\left(\tau,{ }^{w} \tau\right) \neq\{0\}
$$

From (56), we have

$$
I_{J_{r}}\left(\tau,{ }^{w} \tau\right)=J_{r} \cap \widetilde{W}\left(\sigma,{ }^{w} \sigma\right)=J_{r} \cap \widetilde{W}(\sigma) \cdot w=J_{r} \cap D \cdot w .
$$

The result follows from the fact that $J_{r}=I<1, s_{r}>I$.

Inducing the orbit $W_{0} \cdot \alpha$ from $J$ to $J_{1}$ gives 3 distinct elements $\rho_{1}, \phi_{1}, \psi_{1}$, by Lemma 9 . Inducing from $J$ to $L_{0}$ gives $\gamma_{0}$.

Set $C_{2}(\mathfrak{s})=$ free abelian group on the invariant 2 -cycle

$$
\epsilon:=\sum_{w \in W_{0}} \operatorname{sgn}(w)\left({ }^{w} \alpha\right) .
$$

Set $C_{1}(\mathfrak{s})=$ free abelian group on the three invariant 1-cycles

$$
\begin{aligned}
\rho & :=\left(\rho_{1}, \mathcal{R}\left(\rho_{1}\right), \mathcal{R}^{2}\left(\rho_{1}\right)\right), \\
\phi & :=\left(\phi_{1}, \mathcal{R}\left(\phi_{1}\right), \mathcal{R}^{2}\left(\phi_{1}\right)\right), \\
\psi & :=\left(\psi_{1}, \mathcal{R}\left(\psi_{1}\right), \mathcal{R}^{2}\left(\psi_{1}\right)\right) .
\end{aligned}
$$

Set $C_{0}(\mathfrak{s})=$ free vector abelian group on the invariant 0 -cycle

$$
\gamma:=\left(\gamma_{0}, \mathcal{R}\left(\gamma_{0}\right), \mathcal{R}^{2}\left(\gamma_{0}\right)\right)
$$

Note that

$$
\partial\left(\sum_{w \in \operatorname{Alt}(3)}{ }^{w} \alpha\right)=\rho+\phi+\psi
$$


where $\operatorname{Alt}(3)$ is the alternating subgroup of $W_{0}$. Since ${ }^{s_{1} s_{2}} \alpha=\mathcal{R}(\alpha)$, we may also write this as

$$
\partial\left(\alpha+\mathcal{R}(\alpha)+\mathcal{R}^{2}(\alpha)\right)=\rho+\phi+\psi
$$

It follows that $\psi$ is homologous to $-(\rho+\phi)$ in the top row of the double complex $C_{* *}$. This implies that the image of $C(\mathfrak{s})$ in $C_{* *}$ determines 4 homology classes. As representing cycles we may take the 2-cycle $\epsilon$, the two 1-cycles $\rho, \phi$, and the 0 -cycle $\gamma$. Therefore

$$
\mathrm{H}_{\mathrm{ev}}\left(G ; \beta^{1} G\right)^{\mathfrak{s}}=\mathrm{H}_{\text {odd }}\left(G ; \beta^{1} G\right)^{\mathfrak{s}}=\mathbb{Z}^{4} .
$$

Theorem 11. The subspace of the tempered dual of $\mathrm{GL}(3)$ which contains the $\mathfrak{s}$-type $(I, \alpha)$ has the structure of a compact 3-torus. This is a generic torus in the minimal unitary principal series of GL(3). We have

$$
\mathrm{H}_{\mathrm{ev}}\left(G ; \beta^{1} G\right)^{\mathfrak{s}} \cong K_{0}\left(\mathcal{A}^{\mathfrak{s}}\right)=\mathbb{Z}^{4}, \quad \mathrm{H}_{\text {odd }}\left(G ; \beta^{1} G\right)^{\mathfrak{s}} \cong K_{1}\left(\mathcal{A}^{\mathfrak{s}}\right)=\mathbb{Z}^{4}
$$

Proof. Let $\Psi\left(F^{\times}\right)$denote the group of unramified characters of $F^{\times}$. If $\chi \in$ $\Psi\left(F^{\times}\right)$then $\chi(x)=z^{\operatorname{val}(x)}$ with $z$ a complex number of modulus 1 , so that

$$
\Psi\left(F^{\times}\right) \cong \mathbb{T}
$$

Writing

$$
\mathbb{T}^{3}=\left\{\operatorname{Ind}_{T}^{G}\left(\chi_{1} \sigma_{1} \otimes \chi_{2} \sigma_{2} \otimes \chi_{3} \sigma_{3}\right): \chi_{j} \in \Psi\left(F^{\times}\right)\right\}
$$

we have

$$
\mathcal{A}^{\mathfrak{s}} \cong C\left(\mathbb{T}^{3}, \mathfrak{K}\right)
$$

which is strongly Morita equivalent to $C\left(\mathbb{T}^{3}\right)$. The $K$-theory of the 3 -torus is given by

$$
K^{j}\left(\mathbb{T}^{3}\right)=\mathbb{Z}^{4}
$$

where $j=0,1$.

\section{A Chamber homology and K-theory}

Let $G=\mathrm{GL}(N)$ and let $\mathcal{A}$ denote the reduced $C^{*}$-algebra of $G$. Let $\mathcal{H}(G)$ be the convolution algebra of uniformly locally constant, compactly supported, complex-valued functions on $G$, and let $\mathcal{C}(G)$ be the Harish-Chandra Schwartz algebra of $G$. The following diagram serves as a framework for this 
article:

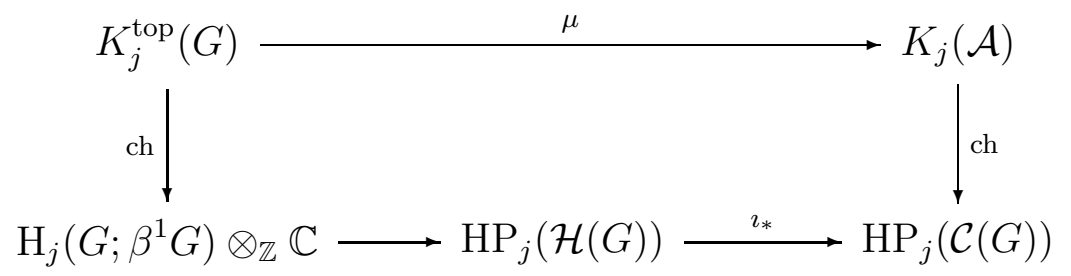

with $j=0,1$. In this diagram, $K_{j}^{\text {top }}(G)$ denotes the topological $K$-theory of $G, K_{j}(\mathcal{A})$ denotes $K$-theory for the $C^{*}$-algebra $\mathcal{A}$. In addition, $\mathrm{HP}_{j}(\mathcal{H}(G))$ denotes periodic cyclic homology of the algebra $\mathcal{H}(G)$, and $\operatorname{HP}_{j}(\mathcal{C}(G))$ denotes periodic cyclic homology of the topological algebra $\mathcal{C}(G)$. For periodic cyclic homology, see [12, 2.4].

The Baum-Connes assembly map $\mu$ is an isomorphism [3, 15]. The map

$$
\mathrm{H}_{*}\left(G ; \beta^{1} G\right) \otimes_{\mathbb{Z}} \mathbb{C} \longrightarrow \mathrm{HP}_{*}(\mathcal{H}(G))
$$

is an isomorphism [14, 20]. The map $\imath_{*}$ is an isomorphism by [3, 6]. The right hand Chern character is constructed in [7] and is an isomorphism after tensoring over $\mathbb{Z}$ with $\mathbb{C}[7$, Theorem 3]. The left hand Chern character is the unique map for which the diagram is commutative.

\section{B The Bernstein spectrum}

Let $G$ be the group of $F$-points of a connected reductive algebraic group defined over $F$. We consider pairs $(L, \sigma)$ where $L$ is a Levi subgroup of a parabolic subgroup of $G$, and $\sigma$ is an irreducible supercuspidal representation of $L$. We say two such pairs $\left(L_{1}, \sigma_{1}\right),\left(L_{2}, \sigma_{2}\right)$ are inertially equivalent if there exist $g \in G$ and an unramified quasicharacter $\chi$ of $L_{2}$ such that

$$
L_{2}=L_{1}^{g} \quad \text { and } \quad \sigma_{1}^{g} \cong \sigma_{2} \otimes \chi .
$$

Here, $L_{1}^{g}:=g^{-1} L_{1} g$ and $\sigma_{1}^{g}(x)=\sigma_{1}\left(g x g^{-1}\right)$ for all $x \in L_{1}^{g}$. We write $[L, \sigma]_{G}$ for the inertial equivalence of the pair $(L, \sigma)$ and $\mathfrak{B}(G)$ for the set of all inertial equivalence classes. The set $\mathfrak{B}(G)$ is the Bernstein spectrum of $G$. We will write $\mathfrak{s} \in \mathfrak{B}(G)$.

The Hecke algebra $\mathcal{H}(G)$ is a unital $\mathcal{H}(G)$-module via left multiplication, and admits the canonical Bernstein decomposition as a purely algebraic direct 
sum of two-sided ideals:

$$
\mathcal{H}(G)=\bigoplus_{\mathfrak{s} \in \mathfrak{B}(G)} \mathcal{H}(G)^{\mathfrak{s}}
$$

This determines the canonical Bernstein decomposition of the reduced $C^{*}$ algebra as a $C^{*}$-direct-sum of two-sided $C^{*}$-ideals:

$$
\mathcal{A}=\bigoplus_{\mathfrak{s} \in \mathfrak{B}(G)} \mathcal{A}^{\mathfrak{s}}
$$

Now $C^{*}$-direct sums are respected by the $K$-theory of $C^{*}$-algebras, and we have

$$
K_{j}(\mathcal{A})=\bigoplus_{\mathfrak{s} \in \mathfrak{B}(G)} K_{j}\left(\mathcal{A}^{\mathfrak{s}}\right)
$$

with $j=0,1$. The abelian groups $K_{j}\left(\mathcal{A}^{\mathfrak{s}}\right)$ are finitely generated free abelian groups, see [17].

We will define $\mathrm{H}_{\text {ev/odd }}\left(G ; \beta^{1} G\right)^{\mathfrak{s}}$ as the pre-image of $K_{j}\left(\mathcal{A}^{\mathfrak{s}}\right)$ via the commutative diagram in Appendix A:

$$
\mathrm{H}_{\mathrm{ev}}\left(G ; \beta^{1} G\right)^{\mathfrak{s}} \cong K_{0}\left(\mathcal{A}^{\mathfrak{s}}\right), \quad \mathrm{H}_{\mathrm{odd}}\left(G ; \beta^{1} G\right)^{\mathfrak{s}} \cong K_{1}\left(\mathcal{A}^{\mathfrak{s}}\right)
$$

\section{The formula for the rank}

Let $\mathfrak{s}$ be a point in the Bernstein spectrum $\mathfrak{B}(G)$, so that $\mathfrak{s}=[L, \sigma]_{G}$. We have

$$
L=\mathrm{GL}\left(m_{1}\right)^{e_{1}} \times \cdots \times \mathrm{GL}\left(m_{r}\right)^{e_{r}}
$$

with $m_{1} e_{1}+\cdots+m_{r} e_{r}=N$. The numbers $e_{1}, \ldots, e_{r}$ are called the exponents of $\mathfrak{s}$, as in [6]. According to [6, Lemma 3.2], we then have

$$
\operatorname{rank} K_{j}\left(\mathcal{A}^{\mathfrak{s}}\right)=2^{r-1} \beta\left(e_{1}\right) \cdots \beta\left(e_{r}\right)
$$

where

$$
\beta(e)=\sum 2^{\kappa(\pi)-1}
$$

In this formula, $\pi$ is a partition of $e$, the sum is over all partitions of $e$, and $\kappa(\pi)$ is the number of unequal parts of $\pi$. For example, if $\pi$ is the partition $1+1+1+3+3+3+3+7+9$ of 31 then $\kappa(\pi)=4$.

The ranks of the finitely generated abelian groups $\mathrm{H}_{\mathrm{ev} / \text { odd }}\left(G ; \beta^{1} G\right)^{\mathfrak{s}}$ are given by

$$
\operatorname{rank} \mathrm{H}_{\text {ev } / \text { odd }}\left(G ; \beta^{1} G\right)^{\mathfrak{s}}=2^{r-1} \beta\left(e_{1}\right) \cdots \beta\left(e_{r}\right) .
$$




\section{Invariants attached to $\mathfrak{s}$}

We write the supercuspidal representation $\sigma$ of the Levi subgroup

$$
M \cong \prod_{i=1}^{q} \prod_{j=1}^{c_{i}} \operatorname{GL}\left(N_{i, j}, F\right)
$$

as a vector $\sigma=\left(\sigma_{1,1}, \ldots, \sigma_{1, c_{1}}, \sigma_{2,1}, \ldots, \sigma_{2, c_{2}}, \ldots, \sigma_{q, 1}, \ldots, \sigma_{q, c_{q}}\right)$ where $\sigma_{i, j}$ is an irreducible supercuspidal representation of $\operatorname{GL}\left(N_{i, j}, F\right)$, and for each $i \in$ $\{1, \ldots, q\}$, the representations $\sigma_{i, j}\left(1 \leq j \leq c_{i}\right)$ admit the same endo-class. At the same time, for all $1 \leq j \leq c_{i}$ and $1 \leq j^{\prime} \leq c_{i^{\prime}}$, the representations $\sigma_{i, j}$ and $\sigma_{i^{\prime}, j^{\prime}}$ have distinct endo-classes if $i^{\prime} \neq i$. This implies that, for a given $i$, in the construction of Bushnell-Kutzko, all the representations $\sigma_{i, j}$ $\left(1 \leq j \leq c_{i}\right)$ may be assumed to correspond to the same field extension $E_{i}$ of $F$. Let $e\left(E_{i} \mid F\right)$ denote the ramification index of $E_{i}$ over $F$. Then the parahoric subgroup $J^{\mathfrak{s}}$ only depends on the integers $N_{i, j}, c_{i}$ and $e\left(E_{i} \mid F\right)$ (see Definition [6).

For supercuspidal representations, the parahoric subgroup is always the same one, say $\operatorname{GL}\left(N, \mathfrak{o}_{F}\right)$; when $q=1$ (that is, only one endo-class), the parahoric is given by the integers $N_{1,1}, \ldots, N_{1, c_{1}}$, which are the sizes of the blocks of $M$. In the general case, the parahoric subgroup depends on the sizes of the blocks of $M$, of the block decomposition defined by the endo-classes (that is, those corresponding to the Levi subgroup $\bar{M} \cong \prod_{i=1}^{q} \operatorname{GL}\left(\bar{N}_{i}\right)$, with $\left.\bar{N}_{i}=\sum_{j=1}^{c_{i}} N_{i, j}\right)$ and on the ramification indices.

\section{References}

[1] Adler, J. and Roche, A.: An intertwining result for $p$-adic groups, Canad. J. Math. 52 (2000) 449-467.

[2] Baum, P., Connes, A. and Higson, N.: Classifying space for proper actions and $K$-theory of group $C^{*}$-algebras, Contemporary Math. 167 (1994) 241-291.

[3] Baum, P., Higson, N. and Plymen, R.J.: A proof of the Baum-Connes conjecture for p-adic GL(n), C. R. Acad. Sci. Paris 325 (1997) 171-176.

[4] Baum, P., Higson, N. and Plymen, R.J.: Representations of p-adic groups: a view from operator algebras, Proc. Symp. Pure Math. 68 (2001) 111-149. 
[5] Bernstein, J. (rédigé par P. Deligne): Le "centre" de Bernstein. Représentations des groupes réductifs sur un corps local. Hermann, Paris (1984) $1-32$.

[6] Brodzki, J. and Plymen, R.J.: Complex structure on the smooth dual of GL(n), Documenta Math. 7 (2002) 91-112.

[7] Brodzki, J. and Plymen, R.J.: Chern character for the Schwartz algebra of p-adic GL(n), Bull. London Math. Soc. 34 (2002) 219-228.

[8] Bushnell, C.J. and Kutzko, P.C.: The admissible dual of GL $(N)$ via compact open subgroups, Annals of Math. Study 129 (1993) Princeton University Press.

[9] Bushnell, C.J. and Kutzko, P.C.: Smooth representations of reductive p-adic groups: structure theory via types, Proc. London Math. Soc. $\mathbf{7 7}$ (1998) 582-634.

[10] Bushnell, C.J. and Kutzko, P.C.: Semisimple types in $\mathrm{GL}_{n}$, Compos. Math. 119 (1999) 53-97.

[11] Bushnell, C.J. and Kutzko, P.C.: Types in reductive $p$-adic groups: the Hecke algebra of a cover, Proc. Amer. Math. Soc. 129 (2001) 601-607.

[12] Cuntz, J., Skandalis, G. and Tsygan, B.: Cyclic homology in noncommutative geometry, EMS 121, Springer-Verlag, Berlin 2004.

[13] Gelfand, S.I. and Manin, Yu. I.: Homological algebra, Springer-Verlag, Berlin 1999.

[14] Higson, N. and Nistor, V.: Cyclic homology of totally disconnected groups acting on buildings, J. Functional Analysis 141 (1996) 466-485.

[15] Lafforgue, V.: K-théorie bivariante pour les algèbres de Banach et conjecture de Baum-Connes, Invent. Math. 149 (2002) 1-95.

[16] Paskunas, V.: Unicity of types for supercuspidal representations of $\mathrm{GL}_{N}$, Proc. London Math. Soc. 91 (2005) 623-654.

[17] Plymen, R.J.: Reduced $C^{*}$-algebra of the $p$-adic group GL(n), J. Functional Analysis 72 (1987) 1-12.

[18] Roche, A.: Types and Hecke algebras for principal series representations of split reductive p-adic groups, Ann. scient. Éc. Norm. Sup. 31 (1998) 361-413. 
[19] Ronan, M.: Lectures on buildings, Academic press (1989).

[20] Schneider, P.: Equivariant homology for totally disconnected groups, J.Algebra 203 (1998) 50-68.

[21] Tits, J.: Reductive groups over local fields, in Automorphic forms, representations and L-functions, Proc. Symp. Pure Math. 33 (1979), part 1, 29-69.

Anne-Marie Aubert, Institut de Mathématiques de Jussieu, U.M.R. 7586 du C.N.R.S., 175 rue du Chevaleret 75013 Paris, France.

Email: aubert@math.jussieu.fr

Samir Hasan, Department of Pure Mathematics, Faculty of Sciences, University of Damascus, Damascus, S.A.R., SYRIA.

Email: samir.hasan@gmail.com

Roger Plymen, School of Mathematics, Manchester University, M13 9PL, England.

Email: plymen@manchester.ac.uk 